\title{
Distinct Subtypes of Somatostatin-Containing Neocortical Interneurons Revealed in Transgenic Mice
}

\author{
Yunyong Ma, ${ }^{1,5}$ Hang Hu, ${ }^{1,5}$ Albert S. Berrebi, ${ }^{1,2,5}$ Peter H. Mathers, ${ }^{2,3,4,5}$ and Ariel Agmon ${ }^{1,5}$ \\ Departments of ${ }^{1}$ Neurobiology and Anatomy, ${ }^{2}$ Otolaryngology, ${ }^{3}$ Biochemistry and ${ }^{4} 0$ phthalmology, and the ${ }^{5}$ Sensory Neuroscience Research Center, West \\ Virginia University, Morgantown, West Virginia 26506
}

\begin{abstract}
GABA-releasing inhibitory interneurons in the cerebral cortex can be classified by their neurochemical content, firing patterns, or axonal targets, to name the most common criteria, but whether classifications using different criteria converge on the same neuronal subtypes, and how many such subtypes exist, is a matter of much current interest and considerable debate. To address these issues, we generated transgenic mice expressing green fluorescent protein (GFP) under control of the GAD67 promoter. In two of these lines, named X94 and $\mathrm{X} 98$, GFP expression in the barrel cortex was restricted to subsets of somatostatin-containing $(\mathrm{SOM}+)$ GABAergic interneurons, similar to the previously reported "GIN" line (Oliva et al., 2000), but the laminar distributions of GFP-expressing (GFP+) cell bodies in the X94, $\mathrm{X} 98$, and GIN lines were distinct and nearly complementary. We compared neurochemical content and axonal distribution patterns of GFP+ neurons among the three lines and analyzed in detail electrophysiological properties in a dataset of 150 neurons recorded in whole-cell, current-clamp mode. By all criteria, there was nearly perfect segregation of X94 and X98 GFP+ neurons, whereas GIN GFP+ neurons exhibited intermediate properties. In the X98 line, GFP expression was found in infragranular, calbindin-containing, layer 1-targeting ("Martinotti") cells that had a propensity to fire low-threshold calcium spikes, whereas X94 GFP + cells were stuttering interneurons with quasi fast-spiking properties, residing in and targeting the thalamo-recipient neocortical layers. We conclude that much of the variability previously attributed to neocortical SOM+ interneurons can be accounted for by their natural grouping into distinct subtypes.
\end{abstract}

Key words: neocortex; GABAergic; somatostatin; transgenic mice; inhibitory interneuron; barrel cortex

\section{Introduction}

Inhibitory, GABA-releasing interneurons are a minority of all cortical neurons but are crucially important for damping cortical excitability, imposing temporal precision on cortical firing, enhancing the saliency of sensory inputs, and promoting longrange synchrony (McBain and Fisahn, 2001; Whittington and Traub, 2003). Moreover, abnormalities in GABAergic neurons are implicated as a major factor in brain disorders ranging from epilepsy to autism and schizophrenia (DeFelipe, 1999; Levitt et al., 2004; Lewis et al., 2005). Such diverse functions are unlikely to be performed by a homogeneous population of neurons. Indeed, as studies during the last two decades have amply revealed, cortical interneurons display highly diverse anatomical, electrophysiological, and molecular properties (Freund and Buzsaki, 1996; Kawaguchi and Kubota, 1997; Markram et al., 2004). Electrophysiologically, interneurons can be described by their firing patterns as fast-spiking (FS), regular-spiking nonpyramidal, lowthreshold spiking (LTS), irregular spiking, or stuttering. Based on

Received July 25, 2005; revised April 3, 2006; accepted April 4, 2006.

This work was supported by National Institutes of Health Grants NS44092 and RR15574. We thank Drs. Kevin Daly, Liset M. de la Prida, and Yael Amitai for helpful comments on this manuscript and Dr. James Culberson for help with histological experiments. Jen Leasa, Ron Fecek, and Cary Johnson provided excellent technical support.

Correspondence should be addressed to Ariel Agmon, Department of Neurobiology and Anatomy, P.0. Box 9128, West Virginia University Health Science Center, Morgantown, WV 26506-9128. E-mail: aric.agmon@gmail.com. DOI:10.1523/JNEUROSCI.0661-06.2006

Copyright $\odot 2006$ Society for Neuroscience $\quad 0270-6474 / 06 / 265069-14 \$ 15.00 / 0$ their axonal targets, soma-preferring (basket), axon-preferring (chandelier), or dendrite-preferring interneurons are recognized. Neurochemically, interneurons may express parvalbumin $(\mathrm{PV})$, somatostatin (SOM), cholecystokinin, or calretinin (CR). Unfortunately, classification schemes based on any individual criterion do not correlate with each other in a simple way, and different categories of properties appear at times to vary independently, leading some researchers to conclude that the number of potential GABAergic subtypes is very high (Gupta et al., 2000) or even that each interneuron is unique (Mott et al., 1997; Parra et al., 1998). This lack of consensus on the classification (and the classifiability) of interneurons has greatly hindered progress on deciphering their role in cortical circuitry (Yuste, 2005).

At the heart of the problem is the uncertainty in identifying the same subsets of neurons in different studies using different techniques. Recently, transgenic mice have been generated in which specific subsets of interneurons belonging to a single neurochemical class express green fluorescent protein (GFP) (Oliva et al., 2000; Meyer et al., 2002; Chattopadhyaya et al., 2004). In a few cases, their detailed analysis revealed novel subtypes within this class. A good example is the PV+ subset, which is probably the best studied neurochemical class of interneurons: initially, all $\mathrm{PV}+$ interneurons were considered fast spiking, but studies using transgenic mice revealed a novel electrophysiological phenotype of bursting PV+ cells (Meyer et al., 2002).

Neocortical SOM+ interneurons appear considerably more 
variable than $\mathrm{PV}+$ interneurons in their neurochemical, morphological, and electrophysiological phenotypes (Kawaguchi and Shindou, 1998; Wang et al., 2004), but so far, no distinct SOM+ subtypes have been described based on a combination of multiple criteria. To use a recently proposed nomenclature (Soltesz, 2005), it is not known whether SOM + interneuron heterogeneity reflects variability (within-group differences) or diversity (the existence of multiple subtypes). Here, we used novel lines of transgenic mice, with GFP-tagged SOM + interneurons, to show that neocortical SOM+ interneurons belong to (at least) two morphologically, neurochemically and electrophysiologically distinct "species," suggesting that SOM+ interneurons may be less variable, but more diverse, than previously recognized.

\section{Materials and Methods}

Generation of GAD67-GFP transgenic mice. The DNA construct used for pronuclear injection was derived from our previously described GAD67GFP vector (Jin et al., 2001, 2003). The vector contained $10.3 \mathrm{~kb}$ of the mouse GAD67 promoter (Szabo et al., 1996; Katarova et al., 1998), including exon 1 , intron 1 , and a small portion of exon 2 , fused in frame at the BamHI site to the enhanced GFP (EGFP) coding region in the pEGFP-1 vector (Clontech, Mountain View, CA). To isolate the fragment for injection using the upstream HindIII site and an AflII site present in the pEGFP-1 vector, we first removed the AflII site present in the GAD67 promoter by end-filling and religating the plasmid. The linearized fragment was injected into fertilized C57BL/6 $\times$ SJL hybrid eggs under contract with DNX (now Xenogen, Cranbury, NJ). Of 11 founder animals positive by PCR, five lines were found to express GFP in the brain and were maintained as hemizygotes by breeding transgenic males with wild-type CD-1 females. Weaned pups were genotyped with GFP primers using standard tail biopsy protocols. GIN mice [strain FVB$\mathrm{Tg}$ (GadGFP) 45704Swn/J] were purchased from The Jackson Laboratory (Bar Harbor, ME); to breed pups for electrophysiological experiments, homozygous GIN males were mated with wild-type CD-1 females.

Immunohistochemistry. For visualization of GFP + neurons, adult mice (2-3 months of age) were anesthetized deeply with $4 \%$ chloral hydrate $(0.02 \mathrm{ml} / \mathrm{g}$, i.p. $)$ and perfused through the ascending aorta with $10 \mathrm{ml}$ of saline, followed by $35 \mathrm{ml}$ of $4 \%$ paraformaldehyde in $0.12 \mathrm{M}$ sodium phosphate buffer (PB), pH 7.3, at a rate of $5 \mathrm{ml} / \mathrm{min}$, using a peristaltic pump. Fixed brains were dissected and postfixed for $4 \mathrm{~h}$ at $4^{\circ} \mathrm{C}$, then placed overnight in $30 \%$ sucrose in $0.075 \mathrm{M} \mathrm{PB}$ at $4^{\circ} \mathrm{C}$ for cryoprotection. Equilibrated brains were sectioned on a freezing microtome (Mikron, San Marcos, CA) into 40- $\mu \mathrm{m}$-thick sections, in either coronal or parasagittal planes, and sections rinsed three times in $0.5 \mathrm{M}$ Tris buffer (Tris), $\mathrm{pH}$ 7.6, at room temperature. To quench endogenous peroxidases, sections were washed for $20 \mathrm{~min}$ in $3 \% \mathrm{H}_{2} \mathrm{O}_{2}$ in $10 \%$ methanol (Sigma, St. Louis, MO), then rinsed three times in Tris and transferred into blocking solution [ $5 \%$ normal goat serum (NGS) plus $0.5 \%$ Triton X-100 (TX) in Tris]. Sections were transferred directly from blocking solution into diluted primary antibody (1:1000 rabbit anti-GFP; Invitrogen, Carlsbad, CA) in vehicle ( $1 \%$ NGS plus $0.1 \%$ TX in Tris) and gently agitated for $36 \mathrm{~h}$ at $4^{\circ} \mathrm{C}$. After three rinses in Tris, sections were transferred into diluted secondary antibody (1:200 biotinylated goat antirabbit; Vector Laboratories, Burlingame, $\mathrm{CA}$ ) in vehicle and rotated for $2 \mathrm{~h}$ at room temperature. After three washes in Tris, sections were incubated in $\mathrm{ABC}$ solution (Vector Laboratories) for $1 \mathrm{~h}$ at room temperature, rinsed three times in Tris, and placed for 3-5 min into $0.05 \% 3,3^{\prime}$ diaminobenzidine tetrahydrochloride (DAB; Sigma) with $0.01 \% \mathrm{H}_{2} \mathrm{O}_{2}$ in Tris. Reaction was stopped with cold buffer, and sections were rinsed three times and mounted from $40 \%$ gelatin-alcohol onto glass slides, air-dried overnight, and coverslipped in a drop of Cytoseal (RichardAllan Scientific, Kalamazoo, MI). DAB-labeled sections were imaged on an Olympus (Melville, NY) Provis AX70 microscope equipped with a Magnafire camera (Optronics, Goleta, CA).

Immunofluorescence. For identification of neurochemical markers, perfusion and sectioning were done as above. Sections were then rinsed three times in PBS, placed in blocking solution (5\% NGS plus 0.5\% TX in
PBS), and rotated for $2 \mathrm{~h}$ at room temperature. Sections were transferred directly from the blocking solution into primary antibody [1:800 rabbit anti-SOM14, T-4103, Bachem Bioscience, King of Prussia, PA; 1:1000 mouse monoclonal anti-PV, Swant, Bellinzona, Switzerland; 1:1000 mouse monoclonal anti-calbindin (anti-CB), Swant; 1:800 rabbit antineuropeptide Y (anti-NPY), T-4070, Bachem] diluted in vehicle solution $\left(1 \% \mathrm{NGS}+0.1 \% \mathrm{TX}\right.$ in PBS) and rotated for $48 \mathrm{~h}$ at $4^{\circ} \mathrm{C}$. After three rinses in PBS, sections were transferred into vehicle with diluted secondary antibody (1:1000 Alexa 546 goat anti-rabbit or anti-mouse; Invitrogen), rotated for $2 \mathrm{~h}$ at room temperature, rinsed three times, mounted in hard-set Vectashield (Vector Laboratories) on glass slides, and coverslipped. Sections were imaged with a Zeiss (Thornwood, NY) LSM510 confocal microscope, using nonsimultaneous excitation of GFP with the $488 \mathrm{~nm}$ line of the argon laser, and of Alexa 546 with the $543 \mathrm{~nm}$ line of the HeNe laser.

Cell counts. To quantify laminar distributions of GFP+ neurons, six coronal sections, from two different rostrocaudal levels through the barrel cortex, were selected for each line. Strips delineating the barrel region were outlined, and all DAB-labeled somatic profiles within each strip were marked under a $10 \times$ objective. Neuroexplorer software (MicroBrightField, Williston, VT) was then used to determine the orthogonal distance between each marked neuron and the pial surface. The fraction of SOM+ interneurons expressing GFP, and vice versa, was estimated from confocal projections of double-labeled sections from barrel cortex.

Electrophysiological recordings. Whole-cell recordings and intracellular staining were performed in juvenile animals [postnatal day 16 (P16) to P24], an age range easily amenable to patch-clamp recordings and comparable with the age used in many previous studies of cortical interneurons in vitro. Cortical inhibitory neurons have acquired many of their adult electrophysiological, morphological and molecular characteristics by the beginning of the third postnatal week (McDonald et al., 1982; Miller, 1986a; Eadie et al., 1987; Minelli et al., 2003; Long et al., 2005). For preparation of brain slices, mice were anesthetized deeply with isoflurane (Abbott Laboratories, Abbott Park, IL) and decapitated. The brains were removed and submerged in ice-cold artificial CSF (ACSF). Using a Vibroslicer (World Precision Instruments, Sarasota, FL), 300- $\mu$ m-thick slices were cut and transferred into a holding chamber filled with recirculated ACSF at room temperature, incubated for at least $1 \mathrm{~h}$, and then transferred to the recording chamber and continuously superfused with $32^{\circ} \mathrm{C}$ ACSF at a rate of $2-3 \mathrm{ml} / \mathrm{min}$. ACSF was saturated with $95 \% \mathrm{O}_{2} / 5 \%$ $\mathrm{CO}_{2}$ and contained the following (in $\mathrm{mM}$ ): $126 \mathrm{NaCl}, 3 \mathrm{KCl}, 1.25$ $\mathrm{NaH}_{2} \mathrm{PO}_{4}, 2 \mathrm{CaCl}_{2}, 1.3 \mathrm{MgSO}_{4}, 26 \mathrm{NaHCO}_{3}$, and 20 D-glucose.

For recording, barrel cortex GFP+ neurons were selected under fluorescence illumination using an Olympus BX50WI microscope equipped with a $40 \times$ water immersion objective and a Hamamatsu (Bridgewater, NJ) Orca CCD camera controlled by SimplePCI software (Compix Imaging Systems, Cranberry Township, PA). The selected cells were then visualized with differential interference contrast optics and targeted by patch pipettes for recordings. Just before seal formation, GFP expression in the targeted cell was reverified by fluorescence. Whole-cell responses were recorded in true current-clamp mode with a patch-clamp amplifier (Axoclamp 2B; Molecular Devices, Sunnyvale, CA). Patch pipettes (5-7 $\mathrm{M} \Omega$ resistance) were pulled from high lead content glass capillaries (PG52165; World Precision Instruments) and filled with intracellular solution containing (in mM): $122 \mathrm{KMeSO}_{4}, 10 \mathrm{KCl}, 2 \mathrm{MgCl}_{2}, 0.1 \mathrm{CaCl}_{2}$, 1.1 EGTA, 10 HEPES, 4 ATP-Mg, 0.3 GTP-Na, and 8 PhosphocreatineTris, pH 7.25, 285-295 mOsm; $2 \mathrm{mg} / \mathrm{ml}$ biocytin (Sigma) was routinely included in the pipette solution. Current pulse protocols were created with the Master-8 pulse generator (A.M.P.I., Jerusalem, Israel); injected current pulses were routinely $600 \mathrm{~ms}$ long. The recorded signals were filtered at $3 \mathrm{kHz}$ and digitized at $10 \mathrm{kHz}$ with a National Instruments (Austin, TX) ADC board. All reported membrane potentials are positively biased by $8 \mathrm{mV}$, which was the measured liquid junction potential in our experiments.

Electrophysiological parameter definitions (in alphabetical order). Afterhyperpolarization (AHP; in millivolts): spike afterhyperpolarization, the difference between threshold and the most negative membrane potential after the spike, measured on the response to the smallest current step evoking an action potential. 
Adaptation ratio (dimensionless): the ratio of $F_{\max }$, steady state to $F_{\max }$, initial.

$F-I$ slope (in hertz/picoampere): frequency-current slope, the slope of the regression line fitted to the initial, steeper portion of the $F-I$ plot. Frequency was defined as the reciprocal of the first interspike interval (ISI).

$F_{\max }$, initial (in hertz): the reciprocal of the first ISI, measured at the maximal current step applied before spike inactivation became evident.

$F_{\max }$, steady state (in hertz): the reciprocal of the average of the last four ISIs, measured at the maximal current step applied before spike inactivation became evident.

$F_{\text {max }}$, stuttering (in hertz): for stuttering cells, the steady-state firing rate at the highest current level at which stuttering was evident (measured as the reciprocal of the average of the last four ISIs during the last firing epoch). For nonstuttering cells, the value of this parameter was defined as zero.

Rate of rise (in volts per second): maximal voltage slope ( $d V / d t$ ) during action potential upswing.

Rate of fall (in volts per second): absolute value of maximal voltage slope $(d V / d t)$ during action potential downswing.

Rheobase (in picoamperes): the intercept of the extrapolated $F-I$ curve with the current axis.

$R_{\text {in }}$ (in megaohms): input resistance, the slope of the regression line fitted to the $V-I$ curve, measured from the response to small current steps resulting in deflections of $\leq 10 \mathrm{mV}$ on both sides of resting potential.

Sag- $V$ slope (dimensionless): the slope of the regression line fitted to the plot of sag versus membrane potential, sag being the difference between the most negative membrane potential during a $600 \mathrm{~ms}$ hyperpolarizing current step and the membrane potential at the end of the step.

Spike height (in millivolts): the difference between threshold and voltage at peak of action potential.

Spike width (in milliseconds): measured at half height between threshold and peak action potential.

Stuttering range (dimensionless): ratio of $F_{\max }$, stuttering to $F_{\max }$, steady state.

$\tau_{m}$ (in milliseconds): membrane time constant, determined from the monoexponential curve best fitting the rising or falling phase of the response to a small hyperpolarizing current step $(\leq 10 \mathrm{mV}$ from resting potential).

Threshold (in millivolts): the membrane potential at the point at which the interpolated rate of rise $(d V / d t)$ of the action potential equaled $5 \mathrm{~V} / \mathrm{s}$, measured on the response to the smallest current step evoking an action potential.

$V_{\text {rest }}$ (in millivolts): resting potential, the stable membrane potential reached a few minutes after breaking the seal, with no current applied.

Morphological reconstruction of recorded neurons. For morphological reconstruction, slices were fixed overnight in $4 \%$ paraformaldehyde in $0.1 \mathrm{M}$ PBS, rinsed with $0.1 \mathrm{M}$ PBS, and permeabilized for $3 \mathrm{~h}$ in $0.6 \%$ Triton X-100 in PBS. To neutralize endogenous peroxidases, slices were pretreated in $1 \% \mathrm{H}_{2} \mathrm{O}_{2}$ in PBS for $30 \mathrm{~min}$. After three additional rinses in $0.1 \mathrm{M}$ PBS, slices were incubated overnight at $4^{\circ} \mathrm{C}$ in $\mathrm{ABC}$ solution in 0.1 м PBS with $0.6 \%$ TX. After three additional rinses in PBS, slices were rinsed for $10 \mathrm{~min}$ in $0.05 \mathrm{M}$ Tris buffer, $\mathrm{pH}$ 7.6, incubated in $0.06 \% \mathrm{DAB}$ in Tris for an additional $15 \mathrm{~min}$, and then the reaction was initiated by adding $0.006 \% \mathrm{H}_{2} \mathrm{O}_{2}$. When the cell body and dendritic processes were clearly visible, the $\mathrm{DAB}$ reaction was stopped with cold PBS solution. Slices were mounted in PBS-glycerol, coverslipped, and sealed with nail polish. Biocytin-filled neurons were visualized, traced, and digitally reconstructed using the Neurolucida system (MicroBrightField) with a $60 \times$ water-immersion objective (Olympus) with a working distance of $\sim 130 \mu \mathrm{m}$.

Multivariate analysis. Multivariate analysis followed Manly (2005). Each cell was represented as a vector in a multidimensional parameter space of $p$ dimensions [ $p$ was 9 for principal component analysis (PCA) and 11 for discriminant function analysis (DFA)]. The full dataset was represented by a "total" matrix $\boldsymbol{T}$ of $r=150$ rows (cells) and $p$ columns (parameters), with values in each column shifted by the grand average of the corresponding parameter so as to have a mean of 0 , and by a similarly sized "within" matrix, $W$, in which the values in each column were shifted independently for each group by the group average of the corresponding parameter. A "between" matrix was defined as $B=T-W$. From $T$, a "normalized total" matrix $N T$ was constructed by scaling each column to have an SD of 1 , and from $W$, a "normalized within" matrix $N W$ was constructed by scaling each group independently to have an SD of 1 .

From $\boldsymbol{T}, \boldsymbol{W}$, and $\boldsymbol{B}$, three $p \times p$ variance-covariance (VCV) matrices were computed (the superscript " $T$ " denotes a transposed matrix) as follows: total VCV matrix, $\boldsymbol{T V}=1 / r\left(\boldsymbol{T}^{\mathrm{T}} \times \boldsymbol{T}\right)$; within (pooled) $\mathrm{VCV}$ matrix, $\boldsymbol{W} \boldsymbol{V}=1 / r\left(\boldsymbol{W}^{\mathrm{T}} \times \boldsymbol{W}\right)$; between VCV matrix, $\boldsymbol{B} \boldsymbol{V}=1 / r\left(\boldsymbol{B}^{\mathrm{T}} \times \boldsymbol{B}\right)$. The within VCV matrix was the average of the three group-wise VCV matrices, weighted by the number of cases in each group. The diagonal elements of $T V, W V$, and $B V$ represented the total, within-group, and between-group variance in each parameter. The fraction of betweengroup variance $\left(\eta^{2}\right)$ for parameter $i$ was therefore $\eta^{2}{ }_{i}=\boldsymbol{B} V_{i, i} / T V_{i, i}$.

From $N T$ and $N W$, two $p \times p$ correlation matrices were computed: total correlation matrix, $T C=1 / r\left(N T^{\mathrm{T}} \times N T\right)$; within (pooled) correlation matrix, $\boldsymbol{W C}=1 / r\left(N W^{\mathrm{T}} \times \boldsymbol{N W}\right)$. The within correlation matrix was the average of the three group-wise correlation matrices, weighted by the number of cases in each group. Matrices TC and $W C$ are shown graphically in Figure 7, $E$ and $F$, respectively. The average correlation within each matrix was defined as the cosine of the average arccosine of the absolute values of the correlation coefficients, calculated over the lower triangle of the matrix.

For PCA, the eigenvalues of WC were calculated, and the two eigenvectors corresponding to the two largest eigenvalues, EVEC1 and EVEC2, used to define the two principal components, by the following: $\mathbf{P C 1}=N T \times$ EVEC1 and PC2 $=N T \times$ EVEC2.

For DFA, the eigenvalues of $\boldsymbol{W} \boldsymbol{V}^{-1} \times \boldsymbol{B V}$ were calculated, and the two eigenvectors corresponding to the two largest eigenvalues, EVEC1 and EVEC2 (not to be confused with the eigenvectors of $\boldsymbol{W C}$ above), used to define the two canonical discriminant functions, by the following: $\mathrm{CDF} 1=T \times \mathrm{EVEC} 1$ and $\mathrm{CDF} 2=T \times \mathrm{EVEC} 2$.

Statistics. Unless noted otherwise, statistical significance values ( $p$ values) were computed numerically, by performing 10,000 random permutations of the dataset and computing the probability for occurrence of values as or more extreme than the experimental result (Good, 1999). Where 10,000 permutations yielded no more extreme values, this is indicated as $p \ll 10^{-4}$. All tests were two-tailed unless noted otherwise. Some data are presented as mean \pm SD.

Software. Data acquisition and processing were done with home-made software written in the LabView environment (National Instruments). Data were tabulated in Excel (Microsoft, Redmond, WA), which was also used to generate scatterplots. Multivariate analysis, correlation matrices, density plots, and permutation tests were programmed in MathCad (MathSoft, Cambridge, MA). Final graphics were prepared with CorelDraw (Corel, Ottawa, Ontario, Canada).

\section{Results}

We generated five transgenic mouse lines with GFP expression under control of the GAD67 promoter. Although all five lines were generated by in ovo injection of the same GAD67-GFP DNA construct (see Materials and Methods), the spatial distribution of GFP + neurons in the brain was distinct in each line. The present study is based on two of these novel transgenic lines, named X94 and X98, in which GFP expression in the cerebral cortex was clearly restricted to nonpyramidal cells, and on the previously published transgenic mouse line GIN in which GFP expression is also driven by the GAD67 promoter, albeit by a shorter segment of the promoter than used by us (Oliva et al., 2000). For convenience, we refer to GFP + neurons in the X94, X98, and GIN lines as "X94 neurons," "X98 neurons," and "GIN neurons," respectively. It should be understood, though, that we consider GFP+ neurons in these mice to be representative of homologous subsets of neurons that are presumed to exist in wild-type animals and, except for GFP expression, to display the same properties. 
Laminar distribution of GFP+ neurons In the neocortex of adult X94, X98, and GIN mice, GFP was expressed only in nonpyramidal, putative GABAergic neurons (Fig. 1). Because there were some interareal variations in the detailed cortical expression patterns within each line, we focused on the somatosensory barrel cortex, which provided an easily identifiable cortical region for interline comparisons and for which the composition and properties of GABAergic interneurons are already known in some detail (Staiger et al., 1996; Porter et al., 2001; Wang et al., 2002, 2004; Beierlein et al., 2003). Qualitative examination of the barrel cortex of adult (2-3 months of age) X94, X98, and GIN mice revealed a distinct laminar distribution pattern of GFP + neurons in each line. X94 neurons were found mostly in layers 4 and $5 \mathrm{~B}$, with a small number in layers $2 / 3,5 \mathrm{~A}$, and upper 6 (Fig. $1 A, D$ ). X98 neurons resided mostly in layers $5 B$ and 6 (Fig. $1 B, E$ ), with a minor population in layers $2 / 3$ (see Fig. $3 B$ ). In the X98 line, low-level expression of GFP was also found in many small cells with glial morphology (faintly visible in Fig. $1 B, E$ ), which formed a remarkably regular lattice and which will not be considered here further. GIN neurons were found mainly in layers $2 / 3$ and $5 \mathrm{~A}$, with a smaller number of cells within layer 4 (Fig. $1 C, F$ ), consistent with their original description (Oliva et al., 2000). Thus, the laminar distributions of $\mathrm{GFP}+$ neurons in the three lines were nearly complementary.

The qualitative impression of complementary distribution patterns was substantiated by counting cells in radial strips through the barrel cortex of the three lines (Fig. $1 A-C$, histograms at the left end of each panel). The interline differences were most striking in layer 4 . In the X94 line, layer 4 contained a high density of GFP-containing neuropil, often segregated into clusters suggestive of barrel boundaries (Fig. 1A,D). Many labeled cell bodies were located at the lower border of layer 4 or within layer $5 \mathrm{~A}$ immediately below this border; layer $5 \mathrm{~A}$ was otherwise very lightly labeled. A second, lower tier of GFP + cell bodies was found in layers 5B (with a small number in upper layer 6). Thus, GFP expression in the X94 cortex closely followed the laminar distribution pattern of thalamocortical afferents (Agmon et al., 1993). In the X98 line, in contrast, GFP expression in layer 4 was totally absent (Fig. $1 \mathrm{~B}$ ). In the GIN line, there was a dense expression of GFP in cell bodies and neuropil immediately above and below layer 4 , but in layer 4 there were only a small number of GFP-containing cell bodies and very little labeled neuropil (Fig. $1 C)$.

Because electrophysiological recordings are often done in brain slices from juvenile mice (as were our recordings), we also examined GFP expression patterns in tissue from 2- to 3-weekold mice (data not shown). GFP expression levels were somewhat lower in juveniles, but expression patterns were overall similar to the adult ones, with a few noteworthy exceptions. In juvenile X98 cortex, strongly labeled GFP + neurons in the supragranular layers were considerably more numerous than in adults. In juvenile GIN mice, there was an apparently ectopic expression of GFP in a subset of pyramidal neurons in the deep cortical layers. GFP expression in 2- to 3-week-old X94 mice was qualitatively very similar to the adult pattern, but in X94 pups younger than 2 weeks. expression was very weak.

\section{Neurochemical characterization of GFP + neurons}

Being nonpyramidal, GFP + neurons in our transgenic lines were, most likely, GABAergic interneurons. Cortical interneurons fall into several distinct neurochemical classes (Kubota et al., 1994; Kawaguchi and Kondo, 2002). To determine whether GFP + neurons in our lines belonged to a specific neurochemical class, we stained sections from adult brains (two animals per line) with antibodies to PV and to SOM (Fig. $2 A-D$ ) and quantified the fraction of GFP+ cells that were also immunopositive for each marker and the fraction of marker immunopositive cells that were also GFP+ (Table 1). Virtually all GFP + neurons in both X94 and X98 lines (95 and 96\%, respectively) were SOM+ (Fig. $2 A, B$ ), and all GFP+ neurons in both lines were PV immunonegative (Fig. $2 C, D$ ) (note that yellow regions in Fig. $2 C$ are the result of overlap of red cell bodies and green neuropil, not colocalization of the labels). We also confirmed that nearly all GIN neurons (97\%) were SOM immunopositive (data not shown), as reported previously (Oliva et al., 2000). GFP+ neurons were clearly only a partial subset of all SOM+ interneurons in their vicinity (Table 1). Even in the layers in which they were most numerous, layers $4,5 / 6$, and $2 / 3$ for X94, X98, and GIN, respectively, GFP + neurons accounted for only 41,20 , and $35 \%$ of all $\mathrm{SOM}+$ neurons, respectively. In total, and assuming that X94, X98, and GIN neurons consist of nonoverlapping populations, GFP + neurons in the three lines accounted for slightly more than one-half of all SOM+ neurons.

Several neurochemicals are known to be expressed in subsets 

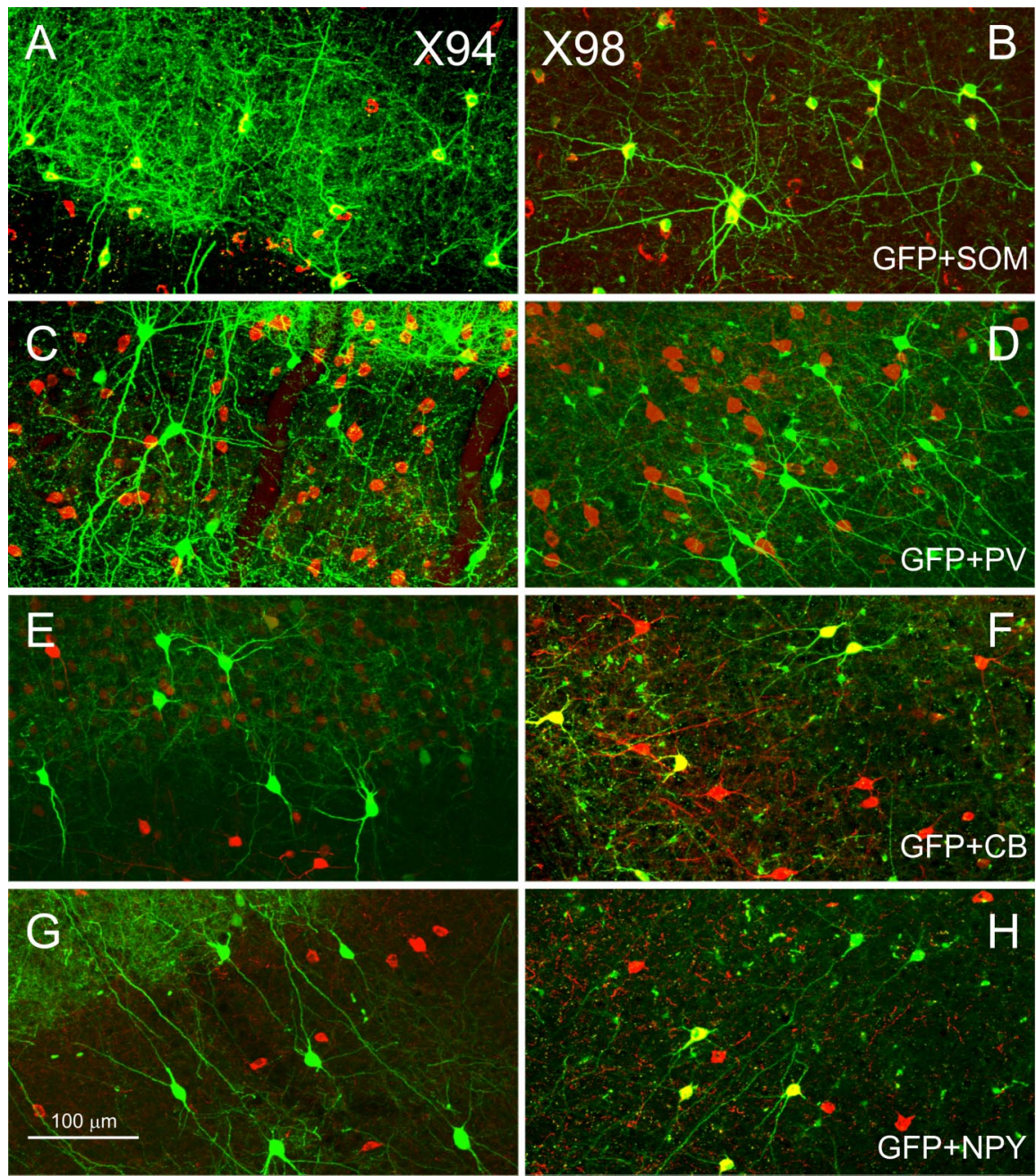

Figure 2. Neurochemical identity of GFP + interneurons. Confocal images of parasagittal sections from X94 $(\boldsymbol{A}, \boldsymbol{C}, \boldsymbol{E}, \boldsymbol{G})$ and $X 98$ $(\boldsymbol{B}, \boldsymbol{D}, \boldsymbol{F}, \boldsymbol{H})$ barrel cortex are shown. GFP fluorescence is pseudocolored green; anti-SOM $(\boldsymbol{A}, \boldsymbol{B})$, anti-PV $(\boldsymbol{C}, \boldsymbol{D})$, anti-CB $(\boldsymbol{E}, \boldsymbol{F})$, and anti-NPY $(\boldsymbol{G}, \boldsymbol{H})$ immunoreactivity is pseudocolored red. Note that in both lines, all GFP + neurons were SOM + and PV - , but only X98 cells were $C B+($ all) and NPY + (some). The yellow seen in C represents overlap in the $z$-dimension between red-labeled $\mathrm{SOM}+$ cell bodies and green-labeled GFP + processes, not colocalization of the two labels.

of SOM + interneurons and could potentially be used as markers for subtypes within the SOM+ population. We tested GFP+ interneurons in our mice for expression of two of these molecules, CB and NPY (two animals per line). X94 neurons never stained for either marker (Fig. $2 E, G$ ). In contrast, virtually all X98 cells (96\%) and about one-third of all GIN cells were immunopositive for CB; $\sim 40 \%$ of all X98 neurons and $27 \%$ of all supragranular GIN cells were immunopositive for NPY (Fig. 2 F,H, Table 1). Thus, X94, X98, and GIN neurons had distinct neurochemical profiles. Moreover, these results indicate that even where they overlap in laminar position, e.g., in layer 5B, X94 and X98 neurons belong to distinct populations.

\section{Axonal morphologies of GFP+ neurons}

Confocal imaging of fixed brain sections from the three lines revealed strong GFP fluorescence in cortical layer 1 of X98 and GIN mice, seemingly emanating from GFP-containing axonal bundles running below the pial surface (Fig. $3 B, C$ ); in contrast, layer 1 in X94 cortex was nearly devoid of fluorescence (Fig. 3A). An axonal projection to layer 1 is the defining feature of Martinotti cells (DeFelipe and Jones, 1988), a morphological class of neocortical interneurons known to express SOM (Wahle, 1993;
Kawaguchi and Kubota, 1996; Shlosberg et al., 2003), but it is not known whether all SOM+ cortical interneurons are Martinotti cells. To further examine this question at a single-cell resolution, we filled $\mathrm{GFP}+$ neurons in the three lines with biocytin during electrophysiological recordings and reconstructed their dendritic and axonal trees using the Neurolucida system. From 230 recorded and filled cells, 69 neurons (35 X94, 19 X98, and 15 GIN cells) retained a sufficiently extensive axonal tree within the slice to warrant a detailed computer-assisted reconstruction; 12 representative reconstructions are illustrated in Figure 4.

In all three lines, the axon (Fig. 4, labeled red) almost always emerged from the upper aspect of the cell body or from an ascending dendritic trunk and, at least initially, extended toward the pial surface. Layer 4 X94 neurons $(n=27)$ (Fig. $4 A-D$ ) had compact axonal trees that remained within layer 4 or (in a few cases) extended into layer 3 and formed a tight cluster that appeared restricted to a single barrel or barrel column. Following the "blueprint" of the barrel cortex (Woolsey et al., 1975; Lorente de Nó, 1992), X94 cells often extended highly asymmetric dendritic and axonal trees toward the presumed center of their barrel (Fig. $4 A-C$ ). In six reconstructed cells, the main axon extended to upper layer $2 / 3$ or even lower layer 1 , where it made a sharp loop and descended back toward layer 4 , in which it made its terminal arbor (Fig. $4 B-D$, turning point indicated by arrows). Only one X94 neuron had an axon that branched within layer 1 (data not shown). Infragranular X94 neurons $(n=8)$ (Fig. $4 E, F)$ emitted a local axonal arbor in the vicinity of the cell body, but in all cases, one or more axonal branches entered layer 4 (and in some cases also layer 3 ) and formed a second arbor there, usually restricted to the same radial column, but in one case (data not shown) extending within layer 4 into adjacent columns. In all cases, by far the most extensive target of X94 neurons was layer 4, never layer 1, confirming the observations from GFP fluorescence (Fig. 3A). Therefore, X94 neurons cannot be considered to be Martinotti cells; rather, they appear to constitute a separate morphological subtype within the SOM+ GABAergic subclass.

X98 axons always ramified immediately above the cell body, usually in upper layer 6 and layer 5. Invariably, however, the main axon continued upwards. This ascending trunk was unfortunately truncated in many cells at the cut surface of the slice, but in the cases in which it was retained within the slice $(n=11)$, it always reached layer 1 . In seven of these cases (examples shown in Fig. $4 J-L)$, the axon ascended as multiple parallel branches through layers 4 and 2/3 before entering layer 1 , where at least some of the branches made a $90^{\circ}$ turn and coursed for some distance parallel to the pial surface, occasionally sending out short terminal side branches. By this pattern of axonal arborizations, which is consistent with the observations of GFP fluores- 
Table 1. Overlap between GFP and SOM, CB, and NPY expression, by line and by laminar position

\begin{tabular}{|c|c|c|c|c|c|c|c|c|c|c|c|c|c|}
\hline \multirow[b]{2}{*}{ Line } & \multirow[b]{2}{*}{ Layer } & \multicolumn{2}{|c|}{$\mathrm{SOM}+$} & \multicolumn{2}{|c|}{ GFP+ } & \multicolumn{2}{|c|}{$\mathrm{CB}+$} & \multicolumn{2}{|c|}{ GFP+ } & \multicolumn{2}{|c|}{ NPY+ } & \multicolumn{2}{|c|}{ GFP+ } \\
\hline & & $n$ & $\% \mathrm{DL}$ & $n$ & $\% \mathrm{DL}$ & $n$ & $\% \mathrm{DL}$ & $n$ & $\% \mathrm{DL}$ & $n$ & $\% \mathrm{DL}$ & $n$ & $\% \mathrm{DL}$ \\
\hline \multirow[t]{4}{*}{ X94 } & $2-3$ & 382 & 5.9 & 23 & 100.0 & & & & & & & & \\
\hline & 4 & 408 & 40.9 & 174 & 96.2 & 48 & 0 & 78 & 0 & 48 & 0 & 30 & 0 \\
\hline & $5-6$ & 1079 & 13.0 & 151 & 92.7 & 425 & 0 & 88 & 0 & 177 & 0 & 65 & 0 \\
\hline & All & 1869 & 17.6 & 348 & 94.9 & 473 & 0 & 166 & 0 & 225 & 0 & 95 & 0 \\
\hline \multirow[t]{4}{*}{ X98 } & $2-3$ & 271 & 12.9 & 35 & 100.0 & 173 & 20.2 & 38 & 92.1 & 126 & 1.6 & 13 & 15.4 \\
\hline & 4 & 188 & 1.7 & 3 & 100 & & & & & & & & \\
\hline & $5-6$ & 694 & 20.3 & 148 & 95.2 & 558 & 21.3 & 124 & 96.0 & 456 & 12.3 & 137 & 40.9 \\
\hline & All & 1153 & 15.6 & 186 & 96.2 & 530 & 20.8 & 115 & 95.7 & 582 & 10.0 & 150 & 38.7 \\
\hline \multirow[t]{4}{*}{ GIN } & $2-3$ & 299 & 34.8 & 110 & 95.9 & 172 & 14.0 & 76 & 31.6 & 291 & 6.2 & 80 & 26.9 \\
\hline & 4 & 171 & 26.5 & 45 & 100 & 40 & 0 & 35 & 0 & 114 & 0 & 71 & 0 \\
\hline & $5-6$ & 492 & 10.8 & 56 & 97.4 & 417 & 11.3 & 96 & 49.0 & 159 & 0.6 & 71 & 1.4 \\
\hline & All & 96 & 21.2 & 211 & 97.1 & 629 & 11.3 & 207 & 34.3 & 564 & 3.4 & 222 & 8.6 \\
\hline
\end{tabular}

For each marker, both the percentage of marker-immunopositive cells expressing GFP and the percentage of GFP-expressing cells immunopositive for the marker are indicated. DL, Double-labeled cells.

cence in layer 1 (Fig. 3B), X98 cells fit the classical definition of Martinotti cells (DeFelipe and Jones, 1988). None of the reconstructed X98 cells in our sample was found to branch within layer 4 to any noticeable degree. Finally, all supragranular GIN cells (examples in Fig. 4G,H), and the one reconstructed layer 5A GIN cell (Fig. $4 I$ ), had multiple ascending axonal branches that branched extensively within layer 1 , consistent with our confocal images (Fig. 3C); GIN neurons were therefore supragranular Martinotti cells.

\section{Firing patterns of GFP + interneurons}

Previous studies reported considerable heterogeneity in the electrophysiological and morphological properties of SOM+ cortical interneurons (Wang et al., 2004), but it remains unclear whether they comprise several distinct subtypes. To address this question, we recorded from GFP+ neurons in the current-clamp, whole-cell mode and compared intracellularly elicited voltage responses between the three lines. This analysis was performed on a dataset of 150 barrel cortex neurons recorded at $32^{\circ} \mathrm{C}$, including 58 X94, 59 X98, and 33 GIN neurons, from 71 juvenile mice (age range, $\mathrm{P} 16-\mathrm{P} 24$; average age, P18.9; no statistical difference in age between lines). All the cells included in this analysis had stable resting potentials more negative than $-60 \mathrm{mV}$ (uncorrected for junction potential) and overshooting action potentials. Although each of the three lines showed some degree of GFP expression in several cortical layers (Fig. 1), we selected our sample from the major concentration of GFP + neurons in each line: layers $4(n=32)$ and $5 \mathrm{~B}(n=26) \mathrm{X} 94$ neurons, infragranular X98 neurons, and supragranular GIN neurons.

When injected with sufficiently large depolarizing current steps, all three lines fired trains of action potentials that exhib-
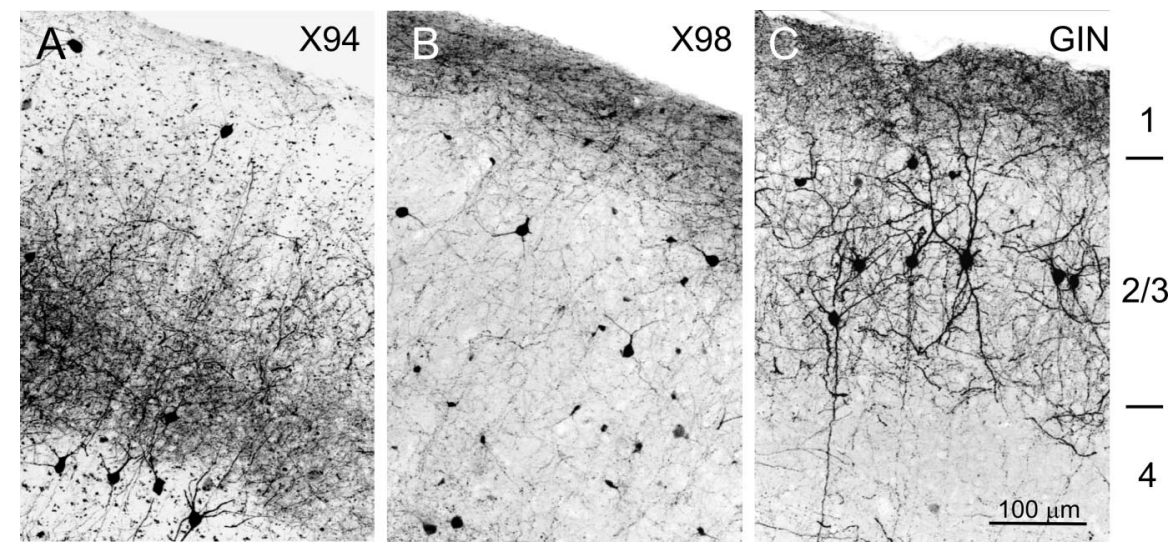

Figure 3. Comparison of axonal projections to layer 1.A-C, Digitally inverted confocal image stacks showing GFP-containing cell bodies and processes in the upper cortical layers of each line. Note the dense band of fluorescent fibers in layer 1 of X98 and GIN, but not X94, cortex.

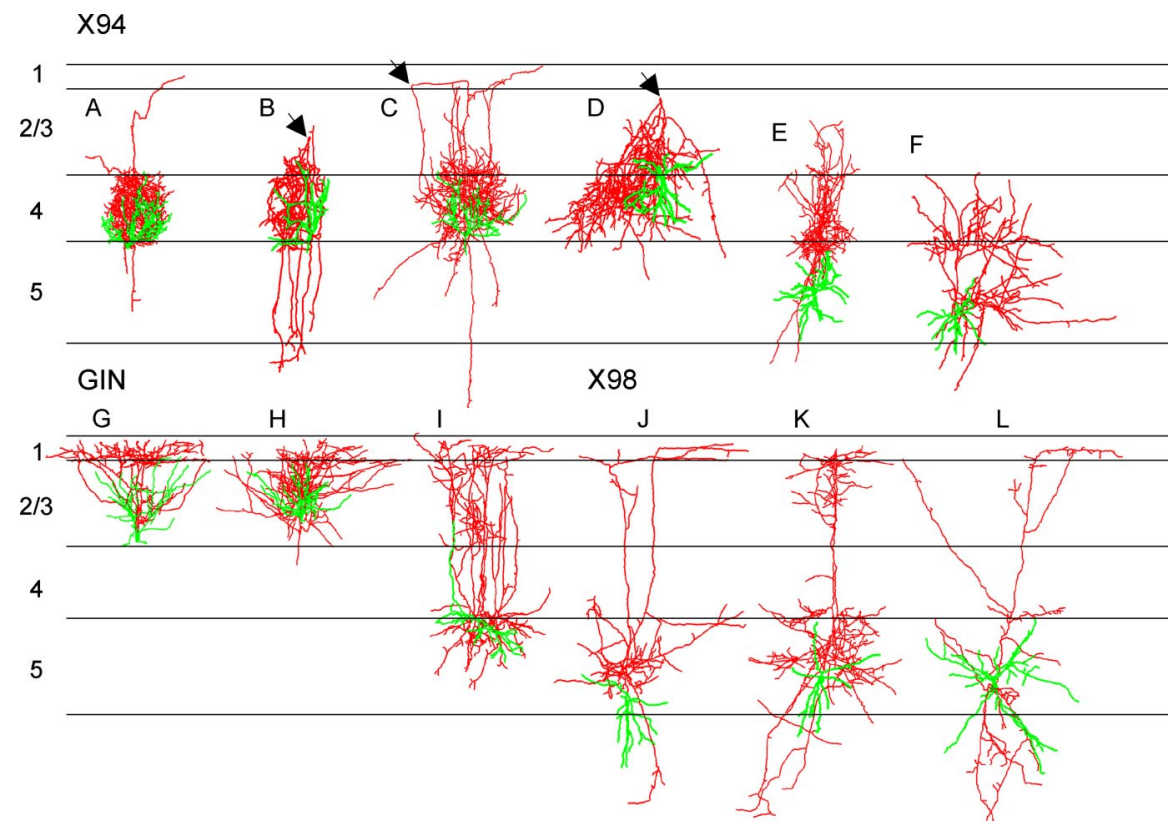

Figure 4. Morphological reconstructions of representative GFP + neurons. $A-L$, Neurons were reconstructed in three dimensions using Neurolucida; cell bodies and dendrites are shown in green, axons in red. For ease of comparison, individual drawings were normalized to the same width of layer 4; average width of layer 4 was $240 \pm 7.5 \mu \mathrm{m}$ (mean \pm SEM). The arrowheads in $\boldsymbol{B}-\boldsymbol{D}$ point to a turning point of the axon, from the upper layers back to layer 4. 


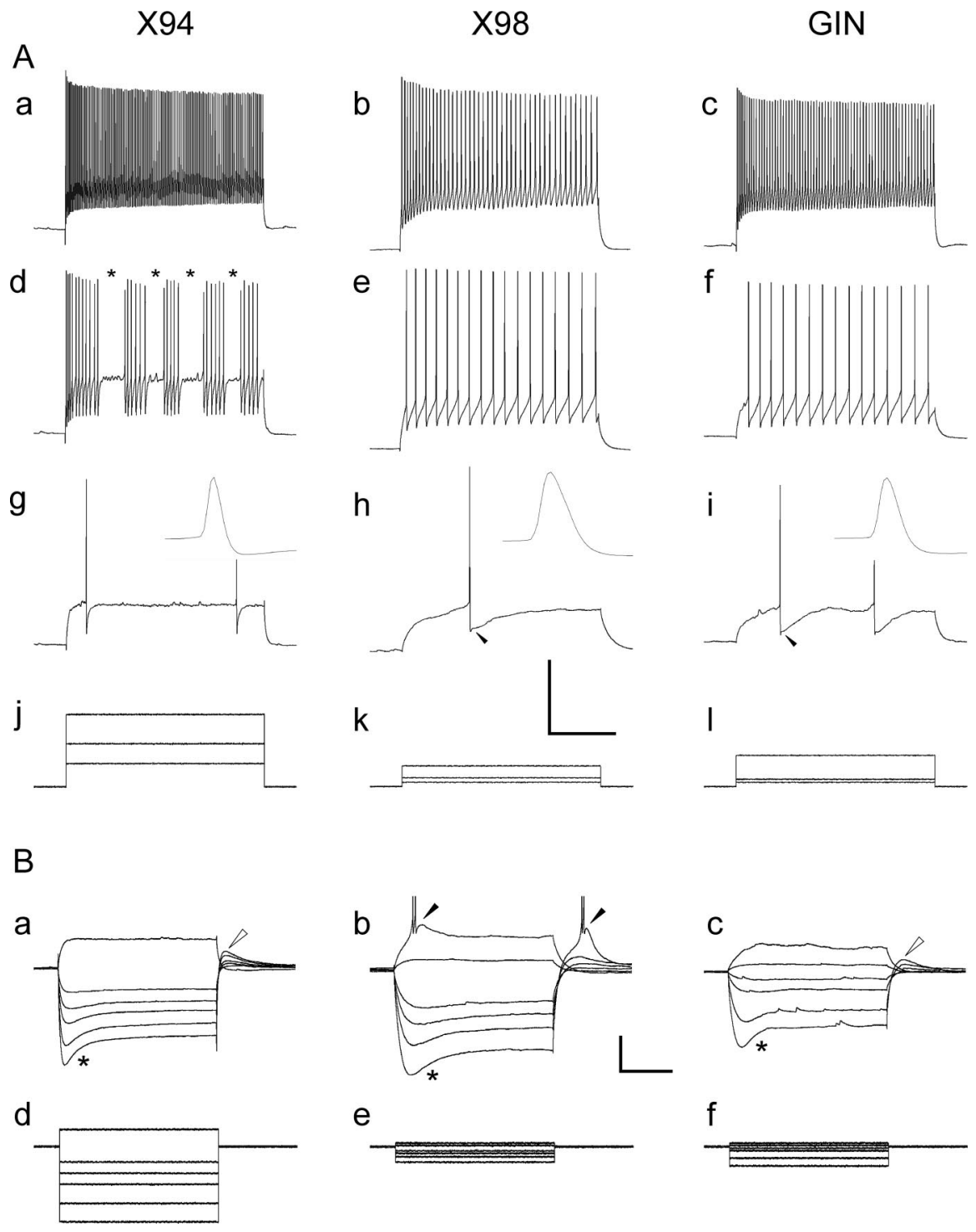

Figure 5. Suprathreshold and subthreshold responses of GFP + neurons. $A$, Spike trains in response to current steps of increasing amplitudes. $\boldsymbol{A a}-\boldsymbol{A c}, \boldsymbol{A d}-\boldsymbol{A f}$, and $\boldsymbol{A g}-\boldsymbol{A i}$ are responses to high, medium, and low current levels, respectively; the three current steps for each neuron are shown superimposed in $\mathbf{A j - A I}$. The asterisks in $\boldsymbol{A d}$ denote interruptions in firing characteristic of stuttering X94 neurons; some GIN neurons also stuttered, but X98 neurons never did. The insets in $\boldsymbol{A g}-\boldsymbol{A i}$ are the first action potential from the corresponding trace, shown at half the vertical scale and at a 100 -fold expanded horizontal scale; note the pronounced difference in spike widths between lines. The arrowheads in $\boldsymbol{A} \boldsymbol{h}$ and $\boldsymbol{A i}$ point to the characteristic triphasic AHP in X98 and GIN neurons. Calibration: $40 \mathrm{mV}(\boldsymbol{A a}-\boldsymbol{A i}), 1000 \mathrm{pA}(\boldsymbol{A j}-\boldsymbol{A})$ ), $200 \mathrm{~ms}$. $\boldsymbol{B}$, Superimposed voltage responses $(\boldsymbol{B a}-\boldsymbol{B C})$ to the hyperpolarizing and small depolarizing current steps shown in $\boldsymbol{B} \boldsymbol{d}-\boldsymbol{B} \boldsymbol{f}$, in three other neurons. Note the very low input resistance of the X94 neuron compared with that of the X98 and GIN neurons (much larger current steps required to elicit similar voltage changes). The asterisks in $\mathbf{B a}-\mathbf{B}$ indicate the sag attributable to the hyperpolarization-activated cationic current $I_{\mathrm{h}}$. The open arrowheads in $\boldsymbol{B} \boldsymbol{a}$ and $\boldsymbol{B} \boldsymbol{c}$ denote a depolarizing rebound, also attributable to $I_{\mathrm{h}}$. The filled arrowheads in $\boldsymbol{B} \boldsymbol{b}$ point to bursts of action potentials riding on low-threshold $\mathrm{Ca}^{2+}$ spikes. Calibration: $20 \mathrm{mV}(\boldsymbol{B a}-\boldsymbol{B C}), 300 \mathrm{pA}(\boldsymbol{B d}-\boldsymbol{B f}), 200 \mathrm{~ms}$.

followed by a sequence of an early fast AHP followed by a delayed "slow" AHP (Zhang and McBain, 1995), which together formed a characteristic triphasic waveform (Fig. 5Ah,Ai, arrowheads). Only $14 \%$ of all X94 cells displayed a triphasic AHP; interestingly, all eight cells that did had input resistances above the median for their group ( $p=0.007$; Fisher's exact test).

X94 neurons were distinctive in another respect; in most cells, spike trains were interrupted at seemingly random intervals for seemingly random periods, often replaced by subthreshold oscillations (Fig. 5Ad, asterisks). Most stuttering cells did so only at the lower range of firing frequencies (Fig. 5, compare $A a, A d$ ), but 11 X94 cells stuttered at all current levels. We quantified the stuttering range of a given cell as the ratio between the highest steady-state firing frequency at which stuttering still occurred to the maximal steady-state firing frequency in the same cell and defined as "robustly stuttering" cells that stuttered through at least the lower one-third of their frequency range (i.e., stuttering range $\geq 0.33$ ). Overall, $58.6 \%$ of X94 cells robustly stuttered, but only $3.3 \%$ of X98 and $12.1 \%$ of GIN neurons did, a highly significant difference between $\mathrm{X} 94$ and the remaining groups $(p=$ $2 \times 10^{-12}$; Fisher's exact test). Layer 4 and layer 5 X94 neurons differed in their propensity to stutter: $75 \%$ of all layer 4 X94 cells robustly stuttered, but only $38.5 \%$ of layer 5 cells did, a highly significant difference between layers ( $p=0.005$; Fisher's exact test). The probability of stuttering was correlated with a low input resistance: $36.7 \%$ of robustly stuttering X94 neurons were in the lower quartile of input resistances for their group, nearly 1.5 times the expected fraction ( $p=0.01$; Fisher's exact test).

In response to hyperpolarizing currents, all three cell groups displayed a sag (Fig. $5 B a-B c$, asterisks), likely to be mediated by the hyperpolarization-activated cationic current $I_{\mathrm{h}}$ (Lupica et al., 2001). All three lines also displayed a depolarizing "rebound" after recovery from the hyper-

ited frequency adaptation. This adaptation was usually most pronounced at the highest current levels, with firing rates at the end of a 600 -ms-long maximal current step falling to $<60 \%$ of the initial rate (Fig. $5 A a-A c$ ). Compared with the other two groups, X94 neurons required much larger current steps to achieve similar voltage deflections (Fig. 5, compare current levels in $A j$ and $B d$ with those in $A k, A l$ and $B e, B f)$, indicative of a much lower input resistance (Table 2) (see below). X94 spikes were pronouncedly narrower than those of X98 and GIN neurons (Fig. $5 A g-A i$, insets, Table 2) (see below). At the lowest firing frequencies, action potentials in $42 \%$ of X98 and $82 \%$ of GIN cells were polarizing step (Fig. 5Ba, $B c$, open arrowheads). In X94 and GIN neurons, this rebound did not trigger spikes, or rarely (in one X94 cell and $18 \%$ of GIN neurons) triggered one action potential. In contrast, in $37 \%$ of X98 neurons recovery from hyperpolarization evoked at least one action potential; $25 \%$ of all X98 cells fired a burst of two to three spikes (Fig. $5 B b$, right filled arrowhead). The difference in rebound spiking probability between X98 cells and the remaining groups was highly significant $\left(p=10^{-5}\right.$; Fisher's exact test). Some X98 cells fired a burst also after depolarization directly from resting potential (Fig. $5 \mathrm{Bb}$, left filled arrowhead). The probability of firing a rebound spike or burst was 
correlated with a high input resistance: $45 \%$ of X98 neurons displaying a rebound spike or burst were in the upper quartile of input resistances for their group, nearly twice the expected fraction ( $p=0.004$; Fisher's exact test). This correlation held also for the X94 and GIN samples: onehalf the GIN neurons that fired a single rebound spike were in the upper quartile of $R_{\text {in }}$ for their group, and the one X94 neuron with a rebound spike had the highest input resistance in its group.

The rebound burst in X98 cells was likely to be triggered by an LTS, mediated by the calcium current $I_{\mathrm{T}}$ (Goldberg et al., 2004). However, the coexistence of a prominent $I_{\mathrm{h}}$ in the same cells raised the possibility that an $I_{\mathrm{h}}$-mediated rebound could have contributed to the bursting in X98 neurons. Several lines of evidence suggested that this was not the case. First, the amplitude of the $I_{\mathrm{h}}$-mediated sag, as measured by the slope of the sag versus voltage curve, was not significantly different between lines (Table 2). Also, there was no significant correlation between sag amplitude and the number of rebound spikes fired by X98 cells $\left(r^{2}=0.03\right)$. To further isolate the contributions of $I_{\mathrm{h}}$ and $I_{\mathrm{T}}$, we used Cs ${ }^{+}$to block $I_{\mathrm{h}}$ channels (Maccaferri and McBain, 1996) and mibefradil to block T-type calcium channels (Yunker, 2003). CsCl (3 mM) totally or nearly totally blocked the sag and the rebound depolarization in X94 and GIN neurons ( $n=4$ cells from each line) (Fig. $6 A, B$, open arrowheads), consistent with both being $I_{\mathrm{h}}$ mediated. $\mathrm{CsCl}$ also strongly blocked the hyperpolarization-induced sag in X98 cells (Fig. 6C, open arrowhead), but in most (five of seven) cells, it did not block the rebound single spike or burst (Fig. 6C, filled arrowhead). In contrast, 10 $\mu \mathrm{M}$ mibefradil had no observable effect on the hyperpolarization-induced sag (Fig. $6 D$, open arrowhead) but totally blocked the rebound burst in two of three cells, even when the hyperpolarizing step was increased (Fig. 6D, filled arrowhead), and reduced the burst to a single spike in the third case. We conclude that X98 cells, but not X94 or GIN neurons, have a propensity to fire low-threshold $\mathrm{Ca}^{2+}$ spikes mediated by $I_{\mathrm{T}}$ and can be classified as LTS cells.

\section{Multivariate analysis of}

electrophysiological parameters

We used the subthreshold and suprathreshold voltage responses to calculate 15 basic electrophysiological parameters for each neuron (Table 2) (see Materials and Methods for definitions of parameters).
Table 2. Values for 15 electrophysiological parameters analyzed for each neuron

\begin{tabular}{|c|c|c|c|c|}
\hline & All $(n=150)$ & X94 $(n=58)$ & X98 $(n=59)$ & $\operatorname{GIN}(n=33)$ \\
\hline \multicolumn{5}{|l|}{$V_{\text {rest }}(m V)$} \\
\hline$\eta^{2}$ & 0.05 & & & \\
\hline$p$ value & 0.0230 & & & \\
\hline $10 \%$ & -72.5 & -73.2 & -72.3 & -69.8 \\
\hline $90 \%$ & -63.9 & -64.6 & -63.7 & -64.7 \\
\hline Mean & -68.3 & -69.1 & -68.2 & -67.1 \\
\hline CV & 0.05 & 0.05 & 0.05 & 0.04 \\
\hline \multicolumn{5}{|c|}{ Threshold (mV) } \\
\hline$\eta^{2}$ & 0.02 & & & \\
\hline$p$ value & 0.2420 & & & \\
\hline $10 \%$ & -47.5 & -47.4 & -48.4 & -47.2 \\
\hline $90 \%$ & -38.3 & -37.9 & -37.4 & -40.5 \\
\hline Mean & -43.0 & -42.8 & -42.6 & -44.0 \\
\hline CV & 0.10 & 0.11 & 0.10 & 0.06 \\
\hline \multicolumn{5}{|c|}{ Spike height (mV) } \\
\hline$\eta^{2}$ & 0.06 & & & \\
\hline$p$ value & 0.0120 & & & \\
\hline $10 \%$ & 51.7 & 51.0 & 53.0 & 48.1 \\
\hline $90 \%$ & 71.8 & 70.1 & 73.5 & 69.3 \\
\hline Mean & 60.9 & 60.3 & 63.0 & 58.1 \\
\hline CV & 0.13 & 0.12 & 0.12 & 0.13 \\
\hline \multicolumn{5}{|c|}{ Sag-V slope } \\
\hline$\eta^{2}$ & 0.01 & & & \\
\hline$p$ value & 0.5240 & & & \\
\hline $10 \%$ & -0.41 & -0.42 & -0.40 & -0.38 \\
\hline $90 \%$ & -0.19 & -0.19 & -0.19 & -0.22 \\
\hline Mean & -0.30 & -0.31 & -0.29 & -0.30 \\
\hline CV & 0.30 & 0.33 & 0.28 & 0.27 \\
\hline \multicolumn{5}{|c|}{ Adaptation ratio } \\
\hline$\eta^{2}$ & 0.04 & & & \\
\hline$p$ value & 0.0620 & & & \\
\hline $10 \%$ & 0.39 & 0.41 & 0.36 & 0.41 \\
\hline $90 \%$ & 0.71 & 0.77 & 0.68 & 0.63 \\
\hline Mean & 0.54 & 0.57 & 0.51 & 0.54 \\
\hline CV & 0.23 & 0.25 & 0.23 & 0.18 \\
\hline \multicolumn{5}{|c|}{ F-I slope (Hz/pA) } \\
\hline$\eta^{2}$ & 0.06 & & & \\
\hline$p$ value & 0.0071 & & & \\
\hline $10 \%$ & 0.47 & 0.53 & 0.40 & 0.55 \\
\hline $90 \%$ & 1.12 & 1.26 & 0.98 & 0.81 \\
\hline Mean & 0.74 & 0.82 & 0.68 & 0.71 \\
\hline CV & 0.35 & 0.34 & 0.39 & 0.25 \\
\hline \multicolumn{5}{|l|}{$R_{\mathrm{in}}(\mathrm{M} \Omega)$} \\
\hline$\eta^{2}$ & 0.55 & & & \\
\hline$p$ value & 0.0000 & & & \\
\hline $10 \%$ & 92 & 73 & 304 & 149 \\
\hline $90 \%$ & 602 & 218 & 796 & 403 \\
\hline Mean & 316 & 132 & 514 & 282 \\
\hline CV & 0.73 & 0.43 & 0.44 & 0.39 \\
\hline \multicolumn{5}{|l|}{$\tau_{m}(\mathrm{~ms})$} \\
\hline$\eta^{2}$ & 0.66 & & & \\
\hline$p$ value & 0.0000 & & & \\
\hline $10 \%$ & 7.2 & 6.0 & 24.1 & 12.5 \\
\hline $90 \%$ & 41.4 & 17.3 & 47.2 & 33.9 \\
\hline Mean & 22.2 & 9.9 & 35.0 & 20.9 \\
\hline CV & 0.62 & 0.42 & 0.29 & 0.41 \\
\hline \multicolumn{5}{|c|}{ Rheobase (pA) } \\
\hline$\eta^{2}$ & 0.63 & & & \\
\hline$p$ value & 0.0000 & & & \\
\hline $10 \%$ & 11 & 77 & 7 & 13 \\
\hline $90 \%$ & 266 & 331 & 57 & 90 \\
\hline Mean & 100 & 202 & 27 & 51 \\
\hline $\mathrm{CV}$ & 1.03 & 0.47 & 0.78 & $\begin{array}{c}0.59 \\
\text { (Table continues) }\end{array}$ \\
\hline
\end{tabular}


Table 2. Continued

\begin{tabular}{|c|c|c|c|c|}
\hline & All $(n=150)$ & $X 94(n=58)$ & $X 98(n=59)$ & $\operatorname{GIN}(n=33)$ \\
\hline \multicolumn{5}{|c|}{ Rate of rise (V/s) } \\
\hline$\eta^{2}$ & 0.38 & & & \\
\hline$p$ value & 0.0000 & & & \\
\hline $10 \%$ & 161 & 217 & 137 & 181 \\
\hline $90 \%$ & 293 & 307 & 241 & 295 \\
\hline Mean & 227 & 261 & 191 & 232 \\
\hline CV & 0.22 & 0.14 & 0.22 & 0.19 \\
\hline \multicolumn{5}{|c|}{ Rate of fall (V/s) } \\
\hline$\eta^{2}$ & 0.59 & & & \\
\hline$p$ value & 0.0000 & & & \\
\hline $10 \%$ & -183 & -201 & -122 & -164 \\
\hline $90 \%$ & -89 & -130 & -79 & -103 \\
\hline Mean & -133 & -166 & -101 & -132 \\
\hline $\mathrm{CV}$ & 0.28 & 0.17 & 0.20 & 0.17 \\
\hline \multicolumn{5}{|c|}{ Spike width (ms) } \\
\hline$\eta^{2}$ & 0.72 & & & \\
\hline$p$ value & 0.0000 & & & \\
\hline $10 \%$ & 0.42 & 0.38 & 0.62 & 0.48 \\
\hline $90 \%$ & 0.79 & 0.50 & 0.86 & 0.63 \\
\hline Mean & 0.58 & 0.45 & 0.74 & 0.55 \\
\hline $\mathrm{CV}$ & 0.27 & 0.13 & 0.16 & 0.10 \\
\hline \multicolumn{5}{|c|}{$F_{\text {max }}$, steady-state $(\mathrm{Hz})$} \\
\hline$\eta^{2}$ & 0.57 & & & \\
\hline$p$ value & 0.0000 & & & \\
\hline $10 \%$ & 49 & 106 & 41 & 80 \\
\hline $90 \%$ & 175 & 201 & 103 & 148 \\
\hline Mean & 113 & 152 & 69 & 120 \\
\hline $\mathrm{CV}$ & 0.44 & 0.26 & 0.33 & 0.26 \\
\hline \multicolumn{5}{|c|}{$F_{\text {max }}$, initial $(\mathrm{Hz})$} \\
\hline$\eta^{2}$ & 0.67 & & & \\
\hline$p$ value & 0.0000 & & & \\
\hline $10 \%$ & 106 & 201 & 86 & 180 \\
\hline $90 \%$ & 304 & 343 & 188 & 265 \\
\hline Mean & 206 & 270 & 135 & 221 \\
\hline CV & 0.36 & 0.19 & 0.27 & 0.18 \\
\hline \multicolumn{5}{|l|}{ AHP (mV) } \\
\hline$\eta^{2}$ & 0.16 & & & \\
\hline$p$ value & 0.0000 & & & \\
\hline $10 \%$ & 13.7 & 13.2 & 15.8 & 13.9 \\
\hline $90 \%$ & 23.3 & 23.0 & 24.1 & 19.8 \\
\hline Mean & 18.3 & 17.5 & 20.2 & 16.5 \\
\hline $\mathrm{CV}$ & 0.21 & 0.23 & 0.18 & 0.15 \\
\hline
\end{tabular}

The 10th and 90 th percentiles, mean, and $C V$ are indicated for each parameter. Also indicated for each parameter are its $\eta^{2}$ and the $p$ value of $\eta^{2}$. The nine parameters in boldface had $\eta^{2}>0.1$ and $p$ values lower than our computational limit of $p=0.0001$ (probably by several orders of magnitude). All parameters are defined in Materials and Methods.

For each parameter, we quantified its overall variance in the sample, by calculating the total sample coefficient of variation (CV; i.e., SD/mean) and its $\eta^{2}$ value (i.e., the fraction of the total variance attributable to differences between, rather than within, the three groups) (see Materials and Methods). When $\eta^{2}$ was plotted against the $\mathrm{CV}$ for each parameter (Fig. 7A), there was a clear clustering of parameters into two groups. Six parameters (Fig. 7A, inside dotted half box) had low between-group variance $\left(\eta^{2}<0.1\right)$ and low to intermediate total variance $(0.05<\mathrm{CV}<$ 0.35 ), and seven parameters (Fig. $7 A$, numbered $1-3,5-8$ ) had high between-group variance $\left(\eta^{2}>0.55\right.$, up to 0.72 for spike width) and intermediate to high total variance $(0.25<\mathrm{CV} \leq 1)$. The remaining two parameters (\#4 and \#9) had intermediate values for both CV and $\eta^{2}$. This correlation between CV and $\eta^{2}$ suggested that most of the variance in the electrophysiological parameters of the neurons in our sample could be accounted for by their natural segregation into three groups.

The six parameters with low $\eta^{2}$ values (resting potential, threshold, spike height, sag-V slope, adaptation ratio, and $F-I$ slope) were clearly not good indicators of group membership and were not analyzed further. The remaining nine parameters had $\eta^{2}>0.1$ (indeed, seven had $\eta^{2} \geq 0.55$ ), values that were very significantly different than 0 $(p \ll 0.0001)$ (Table 2). Thus, these parameters were very good predictors of group membership. Figure $7 B$ shows bygroup density plots for these nine parameters, computed with a Gaussian kernel (Hand et al., 2001). In nearly all plots, X94 and X98 neurons formed clearly separate peaks with very little overlap, whereas GIN neurons occupied intermediate positions, overlapping with both other groups. The clear separation between X94 and X98 can also be appreciated from Table 2: for the seven parameters with $\eta^{2} \geq 0.55$, the $10-$ 90th percentile ranges of the X94 and X98 samples were totally nonoverlapping (see also Table 3). Some of the pairwise differences between means for X98 and X94 parameters were quite large; for example, the average initial and steady-state $F_{\max }$ values were twofold smaller, and the average $R_{\text {in }}$ and $\tau_{m}$ values fourfold larger, in X98 compared with X94.

Given the differences in stuttering range between layer 4 and layer 5 X94 cells, we also compared their electrophysiological parameters. Compared with layer 4 cells, layer 5 X94 neurons had higher $R_{\text {in }}$ $(163 \pm 62$ vs $109 \pm 41 \mathrm{M} \Omega)$, slower $\tau_{m}$ (12.3 \pm 4.8 vs $7.9 \pm 2.1 \mathrm{~ms}$ ), and lower rheobase ( $166 \pm 85$ vs $231 \pm 95 \mathrm{pA})$; these differences were highly significant $(p<$ $0.001, p<0.0001$, and $p<0.01$, respectively). The remaining parameters were not significantly different between the two X94 subgroups.

Given the strong dependence of parameters $1-8$ on group membership (Fig. $7 B)$, we expected them to correlate with each other, as was indeed the case (Fig. $7 C$ ). However, such correlation could also indicate that the parameters in question were not independent (i.e., that they represented different manifestations of the same basic biophysical properties). To identify such intrinsic correlations between parameters, we compared the total sample correlation matrix (Fig. 7C) to the pooled within-group correlation matrix (Fig. 7D) (see Materials and Methods for definition of the two matrices), reasoning that correlations based on shared biophysical mechanisms should be evident also within each group, whereas correlations based solely on cosegregation into different groups should disappear. Indeed, the average correlation coefficient was reduced from 0.58 in the total-sample matrix to 0.34 in the within-group matrix, suggesting that $\sim 60 \%$ of the total sample correlation reflected shared biophysical mechanisms between parameters. Specifically, the within-group correlation matrix (Fig. 7D) suggested that parameters 1-3, representing passive membrane properties, were correlated with each other and that parameters $4-9$, representing action potential 
A $\quad$ X94

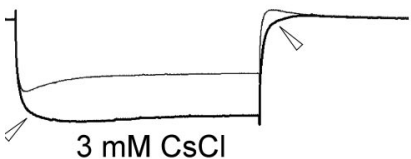

$\bigsqcup$
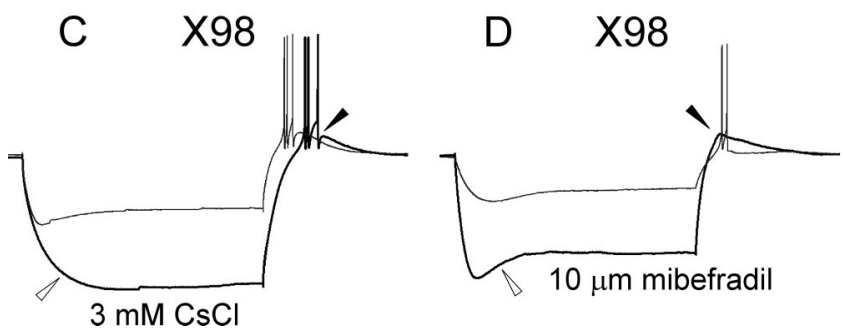

Figure 6. The ionic basis of the rebound burst. In all panels, control traces are drawn as thinner lines. $\mathrm{CsCl}(3 \mathrm{~mm})$, which blocks the hyperpolarization-activated cationic current $I_{\mathrm{h}}$ blocked both the sag and the rebound depolarization in X94 and GIN cells $(\boldsymbol{A}, \boldsymbol{B}$, open arrowheads). In bursting X98 cells, $\mathrm{CsCl}$ blocked the $\operatorname{sag}(\boldsymbol{C}$, open arrowhead) but not the burst (filled arrowhead). In contrast, the $I_{\mathrm{T}}$ channel blocker mibefradil $(10 \mu \mathrm{m})$ did not block the sag $(\boldsymbol{D}$, open arrowhead), but blocked the burst despite the large depolarizing rebound (filled arrowhead) evoked by a stronger hyperpolarization ( $-120 \mathrm{pA}$ in mibefradil, compared with $-40 \mathrm{pA}$ in control). Note that in $\mathbf{A}-\boldsymbol{C}$, the superimposed responses in each panel were evoked by the same current step. Calibration: $40 \mathrm{mV}, 200 \mathrm{~ms}$.

properties, were correlated with each other, but that these two parameter clusters were not correlated with each other. Because information conveyed by intrinsically correlated parameters is redundant, we conducted PCA, a method that reduces such redundancy by redistributing the variance in the dataset among the smallest possible number of independent linear combinations of parameters (Manly, 2005). The two principal components with the largest variances (PC1 and PC2) accounted for 65\% of the total variance in our sample and are plotted against each other in Figure 7E. PC1 and PC2 were correlated, respectively, with the active and passive parameter clusters identified above (correlation coefficients of 0.41 and 0.50 , respectively). In the PC1-PC2 plane, X94 and X98 data points were almost completely segregated, whereas GIN data points occupied the intermediate region and were intermixed, to some degree, with the other two groups. As expected from the differences in passive parameters between layer 4 and layer 5 X94 cells, the latter (Fig. 7E, open red squares) had lower values of PC2, and accounted for most of the overlap between X94 and GIN data points.

PCA maximizes the overall variance per component but does not necessarily improve the discriminability between groups. To determine how well the electrophysiological parameters discriminate between the three subsets, we conducted DFA (see Materials and Methods). This method maximizes the separation between $n$ groups (i.e., maximizes $\eta^{2}$ ) along $n-1$ independent linear combinations of parameters (Manly, 2005). In this analysis, we included the parameters 1-9 above, as well as the stuttering range and the number of rebound spikes fired by each neuron after recovery from a maximal hyperpolarizing step. The resulting two canonical discriminant functions (CDF1 and CDF2) are plotted against each other in Figure 7F. Overall, between-group variance accounted for $87 \%$ of the total variance in CDF1 and for $41 \%$ of the variance in CDF2. Clearly, in the CDF1-CDF2 plane, there was not only a near-perfect separation of X94 and X98 data points along CDF1 but also a very good separation of GIN data points from the other two groups along CDF2. The two intersecting lines in Figure $7 F$ divide the CDF plane into three regions, with X94, X98, and GIN data points mostly segregated into separate regions, indicating that the electrophysiological parameters alone, even without neurochemical or morphological criteria, could be used to classify SOM+ neurons into one of these three groups.

\section{Age-dependent changes in electrophysiological parameters}

Although there were no statistically significant differences in age between the three samples, it was still possible that the large between-group differences we observed were related to differential maturation of cells in different cortical layers and that these differences would disappear by adulthood. This was a priori unlikely, because maturation of cortical layers proceeds in an insideout gradient, whereas electrophysiological properties did not display a pia-to-white matter gradient (e.g., GIN neurons, in the upper layers, had electrophysiological parameters intermediate between X94 cells in the middle layers and X98 cells in the deep layers). Nevertheless, we tested this possibility by performing regression analysis of the two major principal components (PC1 and PC2) against age, by group. PC2 values did not change significantly with age; however, PC1 values showed a weak negative correlation with age, although this effect was statistically significant only in X98 and GIN neurons $\left(r^{2}=0.15\right.$ and 0.16 , respectively). The same effect could be seen in the individual active parameters that correlated with PC1; for example, spikes of X98 neurons narrowed slightly with age, with the trend line dropping by $0.15 \mathrm{~ms}$ through the age range of our experiments. To test how this small age-dependent change in the active parameters may have affected the estimated degree of difference between groups, we divided our sample into three age ranges, $\mathrm{P} 16-\mathrm{P} 17(n=43)$, $\mathrm{P} 18-\mathrm{P} 19(n=59)$, and P20-P24 $(n=48)$, and computed the $\eta^{2}$ values of PC1 separately for each range. $\eta^{2}$ values were $0.78,0.67$, and 0.66 for the three age ranges, respectively, compared with 0.68 for the total sample. We conclude that the contribution of between-group variance to the total variance was somewhat higher at the lower range of ages in our sample but that it stabilized from P18 onwards, probably reaching its mature value, and that the higher $\eta^{2}$ at the younger ages did not bias our conclusions to any significant degree.

\section{Discussion}

We compared two novel and one published transgenic mouse lines in which GFP expression is driven by the GAD67 promoter. Although GAD67 is expressed by all GABAergic interneurons (Mugnaini and Oertel, 1985), cortical GFP expression in all three lines was restricted to the SOM+ neurochemical class. Moreover, each line expressed GFP in a strikingly different subset of SOM+ interneurons, distinct in laminar location, neurochemical markers, axonal morphologies, and electrophysiological properties. Differences in these properties were most pronounced between X94 and X98 neurons, which segregated almost perfectly by all four criteria (Table 3). Although there is no general agreement on what is required to define a neuronal population as a subtype or species (Soltesz, 2005), the extent of the differentially expressed properties we observed approaches the requirements of some recently proposed definitions (Migliore and Shepherd, 2005; Somogyi and Klausberger, 2005) and strongly suggests that at least two of the three subsets (i.e., X94 and X98) belonged to distinct, albeit related, subtypes of SOM+ interneurons.

Putative SOM+ interneurons in the rat were shown previ- 
A
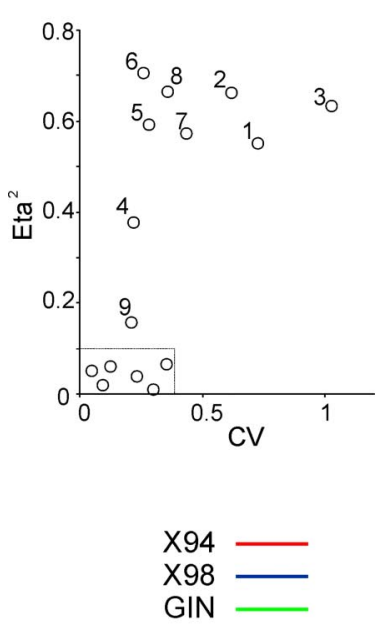

C
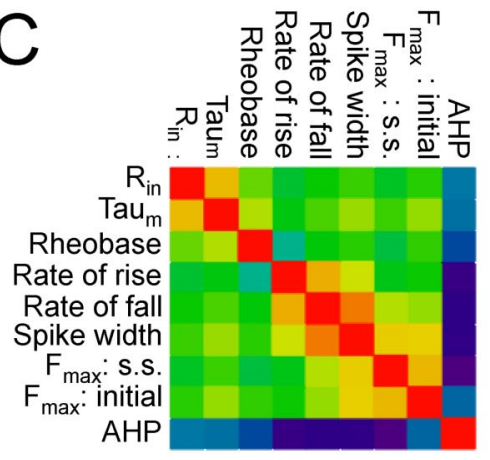

AHP
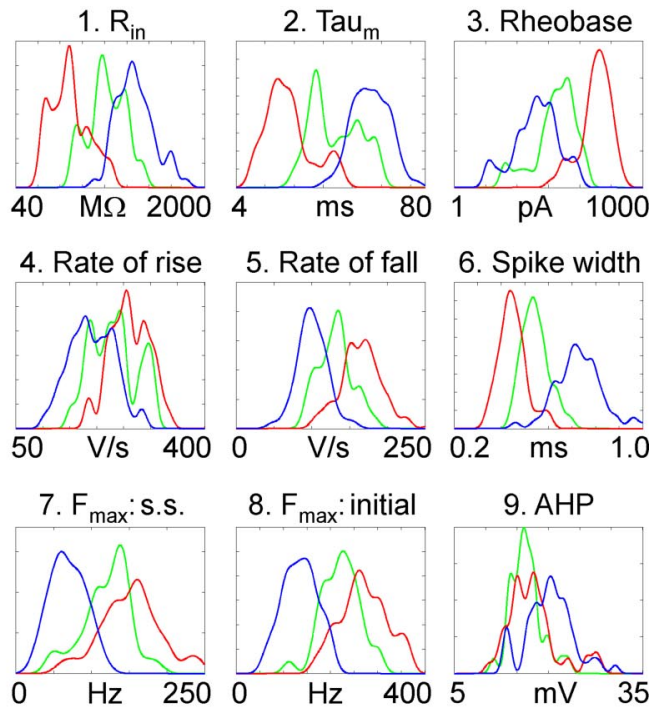
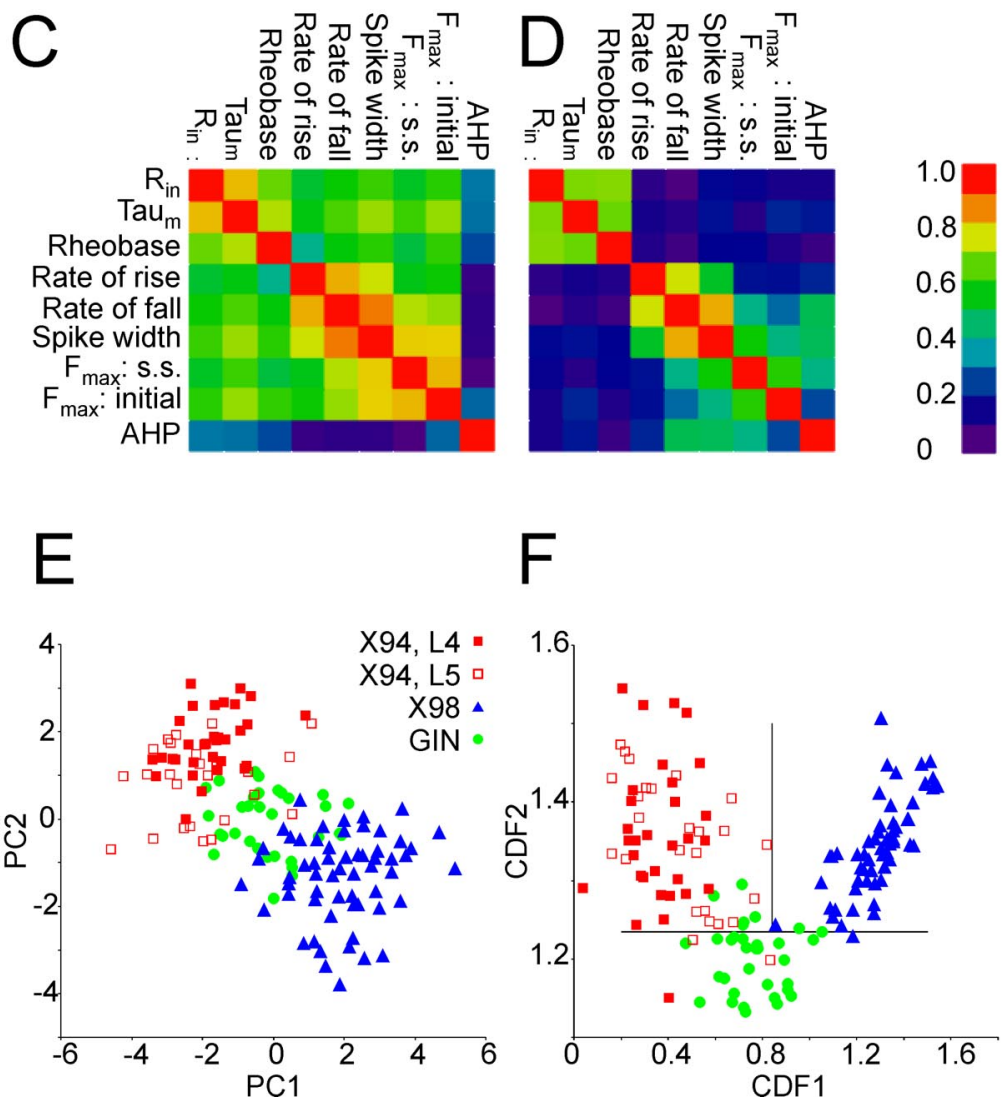

Figure 7. Multivariate analysis of electrophysiological parameters. $A$, Grouping of parameters according to their $C V$ and their $\eta^{2}$. The nine numbered data points correspond, respectively, to the numbered parameters in $\boldsymbol{B}$. The half-box (near the origin of axes) encloses parameters with $\eta^{2}<0.1$ and $C V<0.35$. $B$, Density plots (computed with a Gaussian kernel) of the nine parameters with $\eta^{2}>0.1$, separated by transgenic line. Only the two extreme $x$-values are indicated for each plot. Parameters 1-3 are plotted in a logarithmic scale. $\boldsymbol{C}$, Total-sample correlation matrix of parameters 1-9; the absolute values of the pairwise Pearson correlation coefficients are coded by color. $\boldsymbol{D}$, Pooled within-group correlation matrix of the nine parameters. Note the separate clusters of passive (1-3) and active (4-9) parameters; parameters are correlated within, but not between, each cluster. $\boldsymbol{E}$, Scatterplot of the electrophysiological parameters of the three neuronal subtypes in the principal component plane. Each principal component is a linear combination of the original 9 parameters; $P C 1$ correlates strongly with active parameters, $P C 2$ with passive parameters. $X 94$ data points are separated into layer 4 and layer $5 B$ cells. $\boldsymbol{F}$, Scatterplot of the electrophysiological parameters of the three neuronal subsets in the canonical discriminant function plane. Each CDF is a linear combination of 11 electrophysiological parameters. X94 neurons are separated into layer 4 cells and layer $5 \mathrm{~B}$ cells. The two intersecting lines separate the plane into three regions, with good segregation of the three groups into separate regions. ously to be electrophysiologically, morphologically, and chemically heterogeneous (Wang et al., 2004), but no clear correlations between these different categories of properties were noted. That we observed such correlations is no doubt attributable to our use of transgenic animals, allowing us to target our experiments to well defined and reproducible subsets of neurons identified by genetically encoded vital markers. The identifiability of these subsets means that our results could easily be extended in future studies targeting the same subtypes with additional techniques, e.g., ultrastructural characterization of their synaptic targets (Gulyas et al., 1993; Thomson et al., 1996) or microarray analysis of their gene expression profiles (Lobo et al., 2006; Sugino et al., 2006).

\section{Low-threshold bursts in layer 1-}

\section{targeting, infragranular}

\section{$\mathrm{SOM}+$ interneurons}

X98 neurons resided in the infragranular layers, could generate low-threshold calcium spikes, and sent an ascending projection to layer 1. X98 neurons were therefore similar to LTS Martinotti cells described previously in rodent infragranular cortex (Kawaguchi, 1993, 1995; Deuchars and Thomson, 1995; Goldberg et al., 2004). All X98 cells expressed calbindin, also observed previously in Martinotti neurons (Kawaguchi and Kubota, 1996; Gabbott et al., 1997). As noted previously (Goldberg et al., 2004), SOM + interneurons in supragranular or granular cortical layers never fired low-threshold bursts, and referring (as is often done) to all putative $\mathrm{SOM}+$ interneurons as LTS cells is incorrect.

Low-threshold bursts are thought to be mediated by T-type calcium channels of the $\mathrm{Ca}_{\mathrm{V}} 3 . \mathrm{X}$ gene family (Perez-Reyes, 2003). We confirmed this pharmacologically by showing that the T-channelpreferring blocker mibefradil (Yunker, 2003) blocked the low-threshold bursts. Intriguingly, the occurrence of lowthreshold bursts or single rebound spikes, which were observed in $\sim 40 \%$ of all X98 neurons, correlated with high input resistance. This raises the possibility that a sufficiently high input resistance is required for a cell to generate an LTS and that bursting and nonbursting X98 neurons differed in their input resistance, rather than in the expression of $\mathrm{Ca}_{\mathrm{V}} 3 . \mathrm{X}$ channels.

\section{Stuttering and quasi-fast spiking in} layer 4-targeting SOM+ interneurons X94 cells appear to be a novel subtype of $\mathrm{SOM}+$ interneurons. They fired in a stut- 
Table 3. Summary of the phenotypic differences between X94 and X98 neurons

\begin{tabular}{lll}
\hline Phenotypic property & X94 & X98 \\
\hline Cell body position & Layers 4 and 5B & Layers 5B and 6 \\
Layer 4 axonal arborizations & Dense & None or sparse \\
Layer 1 axonal arborizations & None or sparse & Dense \\
Somatostatin & All & All \\
Calbindin & None & All \\
Neuropeptide Y & None & $\sim 40 \%$ \\
Input resistance & $<250 \mathrm{M} \Omega$ & $>300 \mathrm{MS}$ \\
Time constant & $<20 \mathrm{~ms}$ & $>20 \mathrm{~ms}$ \\
Spike width & $\leq 0.5 \mathrm{~ms}$ & $\geq 0.6 \mathrm{~ms}$ \\
$F_{\text {max }}$ initial & $>200 \mathrm{~Hz}$ & $<200 \mathrm{~Hz}$ \\
$F_{\text {max' }}$ steady-state & $>100 \mathrm{~Hz}$ & $<100 \mathrm{~Hz}$ \\
Robust stuttering & $\sim 60 \%$ & $\sim 3 \%$ \\
Low-threshold spikes & $\sim 2 \%$ & $\sim 40 \%$ \\
\hline
\end{tabular}

Phenotypic properties are arranged in five groups: laminar position, axonal distributions, neurochemical content, electrophysiological parameters, and firing properties. Inequalities in the electrophysiological parameters group apply to at least $90 \%$ of each population.

tering pattern and innervated layer 4 , not layer 1 . The most striking feature of X94 neurons, however, was their electrophysiological parameters, which were within, or very close to, the range of values reported previously for FS interneurons (for example, average spike width of $0.45 \mathrm{~ms}$, compared with $0.31-0.43 \mathrm{~ms}$ for FS cells; average $R_{\text {in }}$ of $132 \mathrm{M} \Omega$, compared with 55-157 M $\Omega$ ) (Kawaguchi, 1995; Galarreta and Hestrin, 2002; Beierlein et al., 2003). The major difference between X94 and FS cells was the pronounced firing frequency adaptation in the former (adaptation ratio of $<0.6$, compared with values of $0.8-1.1$ in FS cells). Thus, X94 cells could be described as "quasi-FS." Why X94-like neurons have not been observed previously in layer 4, where they make up at least $40 \%$ of all SOM + interneurons, is perplexing. It is possible that they are less abundant in the rat, in which most previous studies were conducted (Beierlein et al., 2003; Wang et al., 2004). Alternatively, they could have been encountered but misclassified as FS cells.

Analysis of gene products amplified from single interneurons shows that ion channel genes tend to be expressed in clusters that are characteristic of each neurochemical class (Toledo-Rodriguez et al., 2004). Differential expression of such gene clusters may also underlie the divergence of a given neurochemical class into different subtypes, such as the subtypes of SOM+ interneurons demonstrated here. For example, to account for the unique electrophysiological properties of X94 neurons, the expression of multiple ion channels would be required. The unusually low input resistance of these cells is likely to be caused by "leak" potassium conductances, such as the two-pore channels formed by the KCNK gene family (Goldstein et al., 2001). Stuttering has been associated with the expression of dendrotoxin-sensitive $K_{v} 1.1$ potassium channels (Toledo-Rodriguez et al., 2004), and fastspiking properties are thought to depend on the $\mathrm{K}_{\mathrm{V}} 3$.X family of potassium channels (Erisir et al., 1999; Lien and Jonas, 2003). The detailed molecular basis for the unique electrophysiological fingerprint of X94 cells remains to be determined.

\section{Taxonomy of cortical interneurons}

Despite recent efforts (Yuste, 2005), there is no agreement yet on a system for classification of hippocampal and neocortical interneurons, let alone on a multilevel hierarchy of interneuronal taxa, analogous to grouping of organisms into species, genera, etc. That such groupings do exist, however, has received substantial support from several recent studies (Soltesz, 2005). In molecular systematics, taxonomic distances between organisms are based on the degree of genomic sequence divergence (Sidow and Bowman, 1991); likewise, metrics based on the degree of gene expression similarity could be used for "neuronal systematics." Using such metrics, different neurochemical classes of interneurons in the same cortical region were found to be mutually closer than GABAergic neurons (as a group) were to glutamatergic neurons, but more distant compared with populations of the same neurochemical class in different neocortical regions (Sugino et al., 2006). We expect that subtypes of a single neurochemical class, such as the SOM+ subtypes we described here, would be mutually closer than different neurochemical classes but farther apart than neurons of the same subtype (e.g., X94 neurons) in different cortical areas.

Just as taxonomical separation between biological species reflects phylogenetic distances from a common ancestor, taxonomical separation between neuronal species should reflect ontogenetic distances from a common precursor (Soltesz, 2005). That different neurochemical classes of GABAergic interneurons diverge relatively early in development follows from the findings that they originate from distinct progenitor zones in the ventral telencephalon (Xu et al., 2003, 2004). How then does intraclass diversity arise? Very recently, a fate-mapping study in vivo (Butt et al., 2005) revealed that late-born CR+ interneurons show more phenotypic diversity than early born CR + interneurons, suggesting that intraclass diversity may be generated by temporal, rather than spatial, gradients. Similar experiments have not yet been done on SOM+ interneurons; nevertheless, our finding of distinct subtypes of SOM + interneurons in different cortical layers, together with the pattern of inside-out layering of GABAergic cortical neurons according to age (Miller, 1986b; Valcanis and Tan, 2003), are consistent with a model by which SOM+ interneurons born at different developmental ages are committed to different phenotypes, possibly in response to a changing constellation of transcription factors in the progenitor population (Butt et al., 2005).

The mature phenotype of each interneuron will depend not only on its "genetics," (i.e., the genes it expresses), but also on its local cortical environment. For example, X94 axons seem to respond positively to developmental cues emanating from layer 4 and negatively to cues emanating from layer 1, whereas X98 and GIN axons seem to do the opposite (Katz and Callaway, 1992; Castellani and Bolz, 1997; Castellani et al., 1998). Thus, although the capacity to respond to local molecular cues is likely to be genetically programmed, the detailed morphology of a given neuron is not, accounting for the considerable morphological variability within interneuronal subtypes.

\section{Concluding remarks}

In the past two decades, in vitro electrophysiological studies, together with immunocytochemistry, electron microscopy, computerized morphological reconstruction, and single-cell genomics and proteomics, have greatly expanded the compendium of known properties of neocortical interneurons (Markram et al., 2004). That a unified classification scheme has been slow to emerge is primarily attributable to the inherent uncertainty in the "identity" of cortical neurons, making it difficult to correlate data acquired by different laboratories using different techniques. What has emerged are parallel systems of technique-specific, or even practitioner-specific, classifications, each based on a limited set of features, and which are nearly impossible to reconcile with each other (Soltesz, 2005). The advent of transgenic animals with genetically encoded vital markers has made it possible to identify visually the same subsets of neurons in different animals, provid- 
ing, for the first time, a means to correlate results across techniques, investigators, and studies and integrate them to a holistic portrait of neuronal subtypes, as a necessary step toward the ultimate goal of deciphering the cortical circuit.

\section{References}

Agmon A, Yang LT, O'Dowd DK, Jones EG (1993) Organized growth of thalamocortical axons from the deep tier of terminations into layer IV of developing mouse barrel cortex. J Neurosci 13:5365-5382.

Beierlein M, Gibson JR, Connors BW (2003) Two dynamically distinct inhibitory networks in layer 4 of the neocortex. J Neurophysiol 90:2987-3000.

Butt SJ, Fuccillo M, Nery S, Noctor S, Kriegstein A, Corbin JG, Fishell G (2005) The temporal and spatial origins of cortical interneurons predict their physiological subtype. Neuron 48:591-604.

Castellani V, Bolz J (1997) Membrane-associated molecules regulate the formation of layer-specific cortical circuits. Proc Natl Acad Sci USA 94:7030-7035.

Castellani V, Yue Y, Gao PP, Zhou R, Bolz J (1998) Dual action of a ligand for Eph receptor tyrosine kinases on specific populations of axons during the development of cortical circuits. J Neurosci 18:4663-4672.

Chattopadhyaya B, Di Cristo G, Higashiyama H, Knott GW, Kuhlman SJ, Welker E, Huang ZJ (2004) Experience and activity-dependent maturation of perisomatic GABAergic innervation in primary visual cortex during a postnatal critical period. J Neurosci 24:9598-9611.

DeFelipe J (1999) Chandelier cells and epilepsy. Brain 122:1807-1822.

DeFelipe J, Jones EG (1988) Cajal on the cerebral cortex, pp 457-590. New York: Oxford UP.

Deuchars J, Thomson AM (1995) Innervation of burst firing spiny interneurons by pyramidal cells in deep layers of rat somatomotor cortex: paired intracellular recordings with biocytin filling. Neuroscience 69:739-755.

Eadie LA, Parnavelas JG, Franke E (1987) Development of the ultrastructural features of somatostatin-immunoreactive neurons in the rat visual cortex. J Neurocytol 16:445-459.

Erisir A, Lau D, Rudy B, Leonard CS (1999) Function of specific K(+) channels in sustained high-frequency firing of fast-spiking neocortical interneurons. J Neurophysiol 82:2476-2489.

Freund TF, Buzsaki G (1996) Interneurons of the hippocampus. Hippocampus 6:347-470.

Gabbott PL, Dickie BG, Vaid RR, Headlam AJ, Bacon SJ (1997) Localcircuit neurones in the medial prefrontal cortex (areas 25, 32 and 24b) in the rat: morphology and quantitative distribution. J Comp Neurol 377:465-499.

Galarreta M, Hestrin S (2002) Electrical and chemical synapses among parvalbumin fast-spiking GABAergic interneurons in adult mouse neocortex. Proc Natl Acad Sci USA 99:12438-12443.

Goldberg JH, Lacefield CO, Yuste R (2004) Global dendritic calcium spikes in mouse layer 5 low threshold spiking interneurones: implications for control of pyramidal cell bursting. J Physiol (Lond) 558:465-478.

Goldstein SA, Bockenhauer D, O'Kelly I, Zilberberg N (2001) Potassium leak channels and the KCNK family of two-P-domain subunits. Nat Rev Neurosci 2:175-184.

Good PI (1999) Resampling methods. Boston: Birkhauser.

Gulyas AI, Miles R, Hajos N, Freund TF (1993) Precision and variability in postsynaptic target selection of inhibitory cells in the hippocampal CA3 region. Eur J Neurosci 5:1729-1751.

Gupta A, Wang Y, Markram H (2000) Organizing principles for a diversity of GABAergic interneurons and synapses in the neocortex. Science 287:273-278.

Hand D, Mannila H, Smyth P (2001) Principles of data mining, pp 59-62. Cambridge, MA: MIT.

Jin X, Mathers PH, Szabo G, Katarova Z, Agmon A (2001) Vertical bias in dendritic trees of non-pyramidal neocortical neurons expressing GAD67GFP in vitro. Cereb Cortex 11:666-678.

Jin X, Hu H, Mathers PH, Agmon A (2003) Brain-derived neurotrophic factor mediates activity-dependent dendritic growth in nonpyramidal neocortical interneurons in developing organotypic cultures. J Neurosci 23:5662-5673.

Katarova Z, Mugnaini E, Sekerkova G, Mann JR, Aszodi A, Bosze Z, Greenspan R, Szabo G (1998) Regulation of cell-type specific expression of lacZ by the $5^{\prime}$-flanking region of mouse GAD67 gene in the central nervous system of transgenic mice. Eur J Neurosci 10:989-999.

Katz LC, Callaway EM (1992) Development of local circuits in mammalian visual cortex. Annu Rev Neurosci 15:31-56.

Kawaguchi Y (1993) Groupings of nonpyramidal and pyramidal cells with specific physiological and morphological characteristics in rat frontal cortex. J Neurophysiol 69:416-431.

Kawaguchi Y (1995) Physiological subgroups of nonpyramidal cells with specific morphological characteristics in layer II/III of rat frontal cortex. J Neurosci 15:2638-2655.

Kawaguchi Y, Kondo S (2002) Parvalbumin, somatostatin and cholecystokinin as chemical markers for specific GABAergic interneuron types in the rat frontal cortex. J Neurocytol 31:277-287.

Kawaguchi Y, Kubota Y (1996) Physiological and morphological identification of somatostatin- or vasoactive intestinal polypeptide-containing cells among GABAergic cell subtypes in rat frontal cortex. J Neurosci 16:2701-2715.

Kawaguchi Y, Kubota Y (1997) GABAergic cell subtypes and their synaptic connections in rat frontal cortex. Cereb Cortex 7:476-486.

Kawaguchi Y, Shindou T (1998) Noradrenergic excitation and inhibition of GABAergic cell types in rat frontal cortex. J Neurosci 18:6963-6976.

Kubota Y, Hattori R, Yui Y (1994) Three distinct subpopulations of GABAergic neurons in rat frontal agranular cortex. Brain Res 649:159-173.

Levitt P, Eagleson KL, Powell EM (2004) Regulation of neocortical interneuron development and the implications for neurodevelopmental disorders. Trends Neurosci 27:400-406.

Lewis DA, Hashimoto T, Volk DW (2005) Cortical inhibitory neurons and schizophrenia. Nat Rev Neurosci 6:312-324.

Lien CC, Jonas $P$ (2003) Kv3 potassium conductance is necessary and kinetically optimized for high-frequency action potential generation in hippocampal interneurons. J Neurosci 23:2058-2068.

Lobo MK, Karsten SL, Gray M, Geschwind DH, Yang XW (2006) FACSarray profiling of striatal projection neuron subtypes in juvenile and adult mouse brains. Nat Neurosci 9:443-452.

Long MA, Cruikshank SJ, Jutras MJ, Connors BW (2005) Abrupt maturation of a spike-synchronizing mechanism in neocortex. J Neurosci 25:7309-7316

Lorente de Nó R (1992) The cerebral cortex of the mouse (a first contribution-the "acoustic" cortex). Somatosens Mot Res 9:3-36.

Lupica CR, Bell JA, Hoffman AF, Watson PL (2001) Contribution of the hyperpolarization-activated current $(\mathrm{I}(\mathrm{h}))$ to membrane potential and GABA release in hippocampal interneurons. J Neurophysiol 86:261-268.

Maccaferri G, McBain CJ (1996) The hyperpolarization-activated current (Ih) and its contribution to pacemaker activity in rat CA1 hippocampal stratum oriens-alveus interneurones. J Physiol (Lond) 497:119-130.

Manly BFJ (2005) Multivariate statistical methods: a primer. Boca Raton, FL: Chapman \& Hall/CRC

Markram H, Toledo-Rodriguez M, Wang Y, Gupta A, Silberberg G, Wu C (2004) Interneurons of the neocortical inhibitory system. Nat Rev Neurosci 5:793-807.

McBain CJ, Fisahn A (2001) Interneurons unbound. Nat Rev Neurosci 2:11-23.

McDonald JK, Parnavelas JG, Karamanlidis AN, Brecha N (1982) The morphology and distribution of peptide-containing neurons in the adult and developing visual cortex of the rat. II. Vasoactive intestinal polypeptide. J Neurocytol 11:825-837.

Meyer AH, Katona I, Blatow M, Rozov A, Monyer H (2002) In vivo labeling of parvalbumin-positive interneurons and analysis of electrical coupling in identified neurons. J Neurosci 22:7055-7064.

Migliore M, Shepherd GM (2005) Opinion: an integrated approach to classifying neuronal phenotypes. Nat Rev Neurosci 6:810-818.

Miller MW (1986a) Maturation of rat visual cortex. III. Postnatal morphogenesis and synaptogenesis of local circuit neurons. Brain Res 390:271-285.

Miller MW (1986b) The migration and neurochemical differentiation of gamma-aminobutyric acid (GABA)-immunoreactive neurons in rat visual cortex as demonstrated by a combined immunocytochemicalautoradiographic technique. Brain Res 393:41-46.

Minelli A, Alonso-Nanclares L, Edwards RH, DeFelipe J, Conti F (2003) Postnatal development of the vesicular GABA transporter in rat cerebra cortex. Neuroscience 117:337-346. 
Mott DD, Turner DA, Okazaki MM, Lewis DV (1997) Interneurons of the dentate-hilus border of the rat dentate gyrus: morphological and electrophysiological heterogeneity. J Neurosci 17:3990-4005.

Mugnaini E, Oertel WH (1985) An atlas of the distribution of GABAergic neurons and terminals in the rat CNS as revealed by GAD immunohistochemistry. In: Handbook of chemical neuroanatomy (Bjorklund A, Hokfelt T, eds), Chap 10, pp 436-608. Amsterdam: Elsevier.

Oliva Jr AA, Jiang M, Lam T, Smith KL, Swann JW (2000) Novel hippocampal interneuronal subtypes identified using transgenic mice that express green fluorescent protein in GABAergic interneurons. J Neurosci 20:3354-3368.

Parra P, Gulyas AI, Miles R (1998) How many subtypes of inhibitory cells in the hippocampus? Neuron 20:983-993.

Perez-Reyes E (2003) Molecular physiology of low-voltage-activated t-type calcium channels. Physiol Rev 83:117-161.

Porter JT, Johnson CK, Agmon A (2001) Diverse types of interneurons generate thalamus-evoked feedforward inhibition in the mouse barrel cortex. J Neurosci 21:2699-2710.

Shlosberg D, Patrick SL, Buskila Y, Amitai Y (2003) Inhibitory effect of mouse neocortex layer I on the underlying cellular network. Eur J Neurosci 18:2751-2759.

Sidow A, Bowman BH (1991) Molecular phylogeny. Curr Opin Genet Dev $1: 451-456$

Soltesz I (2005) Diversity in the neuronal machine. New York: Oxford UP.

Somogyi P, Klausberger T (2005) Defined types of cortical interneurone structure space and spike timing in the hippocampus. J Physiol (Lond) 562:9-26

Staiger JF, Zilles K, Freund TF (1996) Distribution of GABAergic elements postsynaptic to ventroposteromedial thalamic projections in layer IV of rat barrel cortex. Eur J Neurosci 8:2273-2285.

Sugino K, Hempel CM, Miller MN, Hattox AM, Shapiro P, Wu C, Huang ZJ, Nelson SB (2006) Molecular taxonomy of major neuronal classes in the adult mouse forebrain. Nat Neurosci 9:99-107.

Szabo G, Katarova Z, Kortvely E, Greenspan RJ, Urban Z (1996) Structure and the promoter region of the mouse gene encoding the $67-\mathrm{kD}$ form of glutamic acid decarboxylase. DNA Cell Biol 15:1081-1091.
Thomson AM, West DC, Hahn J, Deuchars J (1996) Single axon IPSPs elicited in pyramidal cells by three classes of interneurones in slices of rat neocortex. J Physiol (Lond) 496:81-102

Toledo-Rodriguez M, Blumenfeld B, Wu C, Luo J, Attali B, Goodman P, Markram H (2004) Correlation maps allow neuronal electrical properties to be predicted from single-cell gene expression profiles in rat neocortex. Cereb Cortex 14:1310-1327.

Valcanis H, Tan SS (2003) Layer specification of transplanted interneurons in developing mouse neocortex. J Neurosci 23:5113-5122.

Wahle P (1993) Differential regulation of substance P and somatostatin in Martinotti cells of the developing cat visual cortex. J Comp Neuro 329:519-538

Wang Y, Gupta A, Toledo-Rodriguez M, Wu CZ, Markram H (2002) Anatomical, physiological, molecular and circuit properties of nest basket cells in the developing somatosensory cortex. Cereb Cortex 12:395-410.

Wang Y, Toledo-Rodriguez M, Gupta A, Wu C, Silberberg G, Luo J, Markram H (2004) Anatomical, physiological and molecular properties of Martinotti cells in the somatosensory cortex of the juvenile rat. J Physiol (Lond) 561:65-90.

Whittington MA, Traub RD (2003) Interneuron diversity series: inhibitory interneurons and network oscillations in vitro. Trends Neurosci 26:676-682.

Woolsey TA, Dierker ML, Wann DF (1975) Mouse SmI cortex: qualitative and quantitative classification of golgi-impregnated barrel neurons. Proc Natl Acad Sci USA 72:2165-2169.

Xu Q, de la Cruz E, Anderson SA (2003) Cortical interneuron fate determination: diverse sources for distinct subtypes? Cereb Cortex 13:670-676.

Xu Q, Cobos I, De La Cruz E, Rubenstein JL, Anderson SA (2004) Origins of cortical interneuron subtypes. J Neurosci 24:2612-2622.

Yunker AM (2003) Modulation and pharmacology of low voltage-activated (“T-Type") calcium channels. J Bioenerg Biomembr 35:577-598.

Yuste R (2005) Origin and classification of neocortical interneurons. Neuron 48:524-527.

Zhang L, McBain CJ (1995) Potassium conductances underlying repolarization and after-hyperpolarization in rat CA1 hippocampal interneurones. J Physiol (Lond) 488:661-672. 